TRANSACTIONS OF THE

AMERICAN MATHEMATICAL SOCIETY

Volume 357, Number 6, Pages 2165-2186

S 0002-9947(05)03835-3

Article electronically published on January 21, 2005

\title{
STABLE RANK AND REAL RANK FOR SOME CLASSES OF GROUP $C^{*}$-ALGEBRAS
}

\author{
ROBERT J. ARCHBOLD AND EBERHARD KANIUTH
}

\begin{abstract}
We investigate the real and stable rank of the $C^{*}$-algebras of locally compact groups with relatively compact conjugacy classes or finitedimensional irreducible representations. Estimates and formulae are given in terms of the group-theoretic rank.
\end{abstract}

\section{INTRODUCTION}

For a $C^{*}$-algebra $A$, the real $\operatorname{rank} \operatorname{RR}(A)[2]$ and the stable rank $\operatorname{sr}(A)$ [24] have been defined as numerical invariants giving non-commutative analogues of the real and complex dimension of topological spaces. More precisely, for the continuous functions on a compact Hausdorff space $X$ one has $\operatorname{RR}(C(X))=\operatorname{dim} X$ and $\operatorname{sr}(C(X))=\left\lfloor\frac{1}{2} \operatorname{dim} X\right\rfloor+1$, where $\operatorname{dim} X$ is the covering dimension of $X[17,23$. For unital $A$, the stable $\operatorname{rank} \operatorname{sr}(A)$ is either $\infty$ or the smallest possible integer $n$ such that each $n$-tuple in $A^{n}$ can be approximated in norm by $n$-tuples $\left(b_{1}, \ldots, b_{n}\right)$ such that $\sum_{i=1}^{n} b_{i}^{*} b_{i}$ is invertible. Similarly, the real rank $\operatorname{RR}(A)$ is either $\infty$ or the smallest non-negative integer $n$ such that each $(n+1)$-tuple of self-adjoint elements in $A^{n+1}$ can be approximated in norm by $(n+1)$-tuples $\left(b_{0}, b_{1}, \ldots, b_{n}\right)$ of self-adjoint elements such that $\sum_{i=0}^{n} b_{i}^{2}$ is invertible. For non-unital $A$, these ranks are defined to be those of the unitization of $A$. Despite the similarity of these definitions and the fact that $\operatorname{RR}(A) \leq 2 \operatorname{sr}(A)-1$ [2], there are some marked differences between the behaviour of these two ranks. For example, for the algebra of bounded linear operators on an infinite-dimensional Hilbert space $\mathcal{H}$, one has $\operatorname{RR}(\mathcal{B}(\mathcal{H}))=0$ whereas $\operatorname{sr}(\mathcal{B}(\mathcal{H}))=\infty$ 24].

Several authors have computed or estimated the stable and the real rank of group $C^{*}$-algebras $C^{*}(G)$ for various classes of locally compact groups $G[5,6,14,18,25$, 26, 27, 28, 29, 30, 31]. For example, for simply-connected nilpotent Lie groups, Sudo and Takai [30] (following earlier work of Sheu [26]) have shown that $\operatorname{sr}\left(C^{*}(G)\right)$ is the complex dimension of the space of characters of $G$. On the other hand, for the free group $\mathbb{F}_{2}$ on 2 generators it has been shown that $\operatorname{sr}\left(C^{*}\left(\mathbb{F}_{2}\right)\right)=\operatorname{RR}\left(C^{*}\left(\mathbb{F}_{2}\right)\right)=\infty$ [24, 18, but $\operatorname{sr}\left(C_{r}^{*}\left(\mathbb{F}_{2}\right)\right)=\operatorname{RR}\left(C_{r}^{*}\left(\mathbb{F}_{2}\right)\right)=1$ [5] (where $r$ indicates the reduced $C^{*}$-algebra of a non-amenable group). While many $C^{*}$-algebras have real rank zero (e.g. all von Neumann algebras, all approximately finite-dimensional algebras and many simple $C^{*}$-algebras including the Bunce-Deddens and irrational rotation algebras [3]), it is shown in [14 that, for connected $G, \operatorname{RR}\left(C^{*}(G)\right)>0$ if $G$ is not

Received by the editors February 20, 2003.

2000 Mathematics Subject Classification. Primary 46L05, 46L35, 22D25.

This work was supported by a travel grant from the German Research Foundation.

(C)2005 American Mathematical Society Reverts to public domain 28 years from publication 
compact. Similarly, for nilpotent $G, \operatorname{RR}\left(C^{*}(G)\right)>0$ unless every element of $G$ generates a relatively compact subgroup [14].

In this paper we focus on groups with relatively compact conjugacy classes and on groups whose irreducible representations are all finite dimensional (so-called Moore groups). In the first case, we show that the finiteness of $\operatorname{sr}\left(C^{*}(G)\right)$ and $\operatorname{RR}\left(C^{*}(G)\right)$ is equivalent to the finiteness of the rank of the abelian group $G / G^{c}$ (Theorem 2.5). Here $G^{c}$ is the set of all elements in $G$ which generate a relatively compact subgroup ( $G^{c}$ is a closed normal subgroup if $G$ has relatively compact conjugacy classes). A key step in the proof of Theorem 2.5 is an application of Rieffel's theorem that, for any action of $\mathbb{Z}$ on a $C^{*}$-algebra $A, \operatorname{sr}\left(A \times_{\alpha} \mathbb{Z}\right) \leq \operatorname{sr} A+1$ 24, Theorem 7.1]. Under an additional hypothesis, we obtain in Theorem 2.6 the formulas $\operatorname{sr}\left(C^{*}(G)\right)=\left\lfloor\frac{n}{2}\right\rfloor+1$ and $\operatorname{RR}\left(C^{*}(G)\right)=n$ if $G / G^{c}$ has finite rank $n$.

For a Moore group $G$, the ranks of $C^{*}(G)$ are related to the rank of the subgroup $G_{F}$ consisting of all elements of $G$ with relatively compact conjugacy classes. To be precise, we show in Theorem 3.4 that

$$
\left\lceil\frac{\operatorname{rank}\left(G_{F}\right)}{2\left[G: G_{F}\right]-1}\right\rceil \leq \operatorname{RR}\left(C^{*}(G)\right) \leq \operatorname{rank}\left(G_{F}\right),
$$

and that a result of a similar nature holds for $\operatorname{sr}\left(C^{*}(G)\right)$. In particular, the finiteness of $\operatorname{sr}\left(C^{*}(G)\right)$ and $\operatorname{RR}\left(C^{*}(G)\right)$ is equivalent to the finiteness of the rank of $G_{F}$. We then characterize the Moore groups $G$ for which $\operatorname{RR}\left(C^{*}(G)\right)=\operatorname{rank}\left(G_{F}\right)$ and also those for which $\operatorname{sr}\left(C^{*}(G)\right)$ attains the corresponding upper bound (Theorems 4.3 and 4.4). Section 4 concludes with some examples.

\section{Stable And Real Rank of SOME type I $C^{*}$-Algebras}

We note here, for use in this and later sections, that if $J$ is a closed (two-sided) ideal of a $C^{*}$-algebra $A$, then $\operatorname{sr}(A / J), \operatorname{sr}(J) \leq \operatorname{sr}(A)$ [24, Section 4] and similarly for the real rank [8, Théorème 1.4]. Recall that, for a non-negative real number $x$, $\lfloor x\rfloor$ is the greatest integer $n$ such that $n \leq x$ and $\lceil x\rceil$ is the least integer $m$ such that $m \geq x$. Since the covering dimension $\operatorname{dim} X$ takes values in $\mathbb{N} \cup\{\infty\},\lfloor\infty\rfloor$ and $\lceil\infty\rceil$ should be understood as $\infty$. The following results will be required in the subsequent sections.

Lemma 1.1. (a) Let $A$ be an $n$-homogeneous $C^{*}$-algebra with compact spectrum $\widehat{A}$. Then

(i) $\operatorname{RR}(A)=\left\lceil\frac{\operatorname{dim} \hat{A}}{2 n-1}\right\rceil$.

(ii) $\operatorname{sr}(A)=\left\lceil\frac{1}{n}\left\lfloor\frac{1}{2} \operatorname{dim} \widehat{A}\right\rfloor\right\rceil+1$.

(b) Let $A$ be a separable $C^{*}$-algebra with continuous trace such that $\operatorname{dim} \pi=\aleph_{0}$ for all $\pi \in \widehat{A}$ and such that $\widehat{A}$ is compact and has finite dimension. Then

(i) $\operatorname{RR}(A)=\min \{1, \operatorname{dim} \widehat{A}\}$.

(ii) $\operatorname{sr}(A)=\min \left\{2,1+\left\lfloor\frac{1}{2} \operatorname{dim} \widehat{A}\right\rfloor\right\}$.

Proof. (a) By local triviality [32], for each $t \in \widehat{A}$ there exists an open neighbourhood $U_{t}$ such that $A / J_{t} \cong C\left(\overline{U_{t}}\right) \otimes M_{n}$, where $J_{t}$ is the closed ideal of $A$ such that $\widehat{J}_{t}=\widehat{A} \backslash \overline{U_{t}}$. By compactness, $\widehat{A}$ is covered by a finite number of such open sets, 
$U_{1}, \ldots, U_{m}$ say. With $J_{i}$ defined by $\widehat{J}_{i}=\widehat{A} \backslash \overline{U_{i}}(1 \leq i \leq m)$, we have

$$
\bigcap_{i=1}^{m} \widehat{J}_{i}=\widehat{A} \backslash \bigcup_{i=1}^{m} \overline{U_{i}}=\emptyset
$$

and so $\bigcap_{i=1}^{m} J_{i}=\{0\}$.

(i) Since $\operatorname{RR}\left(A / J_{i}\right) \leq \operatorname{RR}(A)(1 \leq i \leq m)$, we obtain from [22, Lemma 1.7] and [19, Proposition 1.6] that

$$
\operatorname{RR}(A)=\max _{i \leq i \leq m} \operatorname{RR}\left(A / J_{i}\right)=\max _{1 \leq i \leq m} \operatorname{RR}\left(C\left(\overline{U_{i}}\right) \otimes M_{n}\right)=\max _{1 \leq i \leq m}\left\lceil\frac{\operatorname{dim} \overline{U_{i}}}{2 n-1}\right\rceil,
$$

where the last equality follows from [1, Corollary 3.2]. Since $\widehat{A}$ is normal, by the (countable) sum theorem [23, Chapter 3, Theorem 2.5],

$$
\operatorname{dim} \widehat{A}=\max _{1 \leq i \leq m} \operatorname{dim} \overline{U_{i}} .
$$

Equations (1) and (2) yield the required result.

(ii) Since $\operatorname{sr}\left(A / J_{i}\right) \leq \operatorname{sr}(A)(1 \leq i \leq m)$, we obtain from [20, Corollary 2.7] that

$$
\begin{aligned}
\operatorname{sr}(A) & =\max _{1 \leq i \leq m} \operatorname{sr}\left(A / J_{i}\right)=\max _{1 \leq i \leq m} \operatorname{sr}\left(C\left(\overline{U_{i}}\right) \otimes M_{n}\right) \\
& =\max _{1 \leq i \leq m}\left\lceil\frac{1}{n}\left\lfloor\frac{1}{2} \operatorname{dim} \overline{U_{i}}\right\rfloor \mid+1,\right.
\end{aligned}
$$

where the last equality follows from [24]. Equation (2) yields the required result.

(b) By local triviality [5, (3.3.3) and (10.9.5)(i)], for each $t \in \widehat{A}$ there exists an open neighbourhood $U_{t}$ such that $A / J_{t} \cong C\left(\overline{U_{t}}\right) \otimes \mathcal{K}$, where $J_{t}$ is the closed ideal of $A$ such that $\widehat{J}_{t}=\widehat{A} \backslash \overline{U_{t}}$ and $\mathcal{K}$ is the $C^{*}$-algebra of compact operators on a separable Hilbert space of infinite dimension. Note that if $X$ is a compact Hausdorff space and $\operatorname{dim} X \geq 1$, then $\operatorname{RR}(C(X) \otimes \mathcal{K})=1$ by [1] Proposition 3.3 et seq.], but if $\operatorname{dim} X=0$, then $\operatorname{RR}(C(X) \otimes \mathcal{K})=0$ by [2, Corollary 3.3]. Hence, proceeding as in (a), we obtain that

$$
\operatorname{RR}(A)=\max _{1 \leq i \leq m}\left(C\left(\overline{U_{i}}\right) \otimes \mathcal{K}\right)=\max _{1 \leq i \leq m} \min \left\{1, \operatorname{dim} \overline{U_{i}}\right\}=\min \{1, \operatorname{dim} \widehat{A}\} .
$$

Similarly, using [24, Theorem 6.4 and Proposition 1.7],

$$
\begin{aligned}
\operatorname{sr}(A) & =\max _{1 \leq i \leq m} \operatorname{sr}\left(C\left(\overline{U_{i}}\right) \otimes \mathcal{K}\right)=\max _{1 \leq i \leq m} \min \left\{2,1+\left\lfloor\frac{1}{2} \operatorname{dim} \overline{U_{i}}\right\rfloor\right\} \\
& =\min \left\{2,1+\left\lfloor\frac{1}{2} \operatorname{dim} \widehat{A}\right\rfloor\right\} .
\end{aligned}
$$

Proposition 1.2. Let $A$ be a $C^{*}$-algebra with continuous trace and let $\mathcal{C}$ denote the set of all compact subsets of $\widehat{A}$.

(a) Suppose that $A$ is n-homogeneous. Then

(i) $\operatorname{RR}(A)=\sup _{C \in \mathcal{C}}\left\lceil\frac{\operatorname{dim} C}{2 n-1}\right\rceil \leq\left\lceil\frac{\operatorname{dim} \hat{A}}{2 n-1}\right\rceil$.

(ii) $\operatorname{sr}(A)=\sup _{C \in \mathcal{C}}\left\lceil\frac{1}{n}\left\lfloor\frac{1}{2} \operatorname{dim} C\right\rfloor\right\rceil+1 \leq\left[\frac{1}{n}\left\lfloor\frac{1}{2} \operatorname{dim} \widehat{A}\right\rfloor\right\rceil+1$. 
(b) Suppose that $A$ is separable, that $\operatorname{dim} \pi=\aleph_{0}$ for all $\pi \in \widehat{A}$ and that $\operatorname{dim} \widehat{A}<$ $\infty$. Then
(i) $\operatorname{RR}(A)=\min \left\{1, \sup _{C \in \mathcal{C}} \operatorname{dim} C\right\} \leq \min \{1, \operatorname{dim} \widehat{A}\}$.
(ii) $\operatorname{sr}(A)=\min \left\{2,1+\left\lfloor\frac{1}{2} \sup _{C \in \mathcal{C}} \operatorname{dim} C\right\rfloor\right\} \leq \min \left\{2,1+\left\lfloor\frac{1}{2} \operatorname{dim} \widehat{A}\right\rfloor\right\}$.

Proof. Since $\widehat{A}$ is Hausdorff, each $C \in \mathcal{C}$ is closed and so $\operatorname{dim} C \leq \operatorname{dim} \widehat{A}[23$. Chapter 3, Proposition 1.5]. For each open set $W$ in $\widehat{A}$, let $J_{W}$ be the closed ideal of $A$ such that $\widehat{J_{W}}=W$.

(a) Let $C \in \mathcal{C}$. Then $A / J_{(\widehat{A} \backslash C)}$ is $n$-homogeneous with spectrum $C$ and so, by Lemma 1.1(a),

$$
\operatorname{RR}(A) \geq \operatorname{RR}\left(A / J_{(\widehat{A} \backslash C)}\right)=\left\lceil\frac{\operatorname{dim} C}{2 n-1}\right\rceil
$$

and

$$
\operatorname{sr}(A) \geq \operatorname{sr}\left(A / J_{(\widehat{A} \backslash C)}\right)=\left\lceil\frac{1}{n}\left\lfloor\frac{1}{2} \operatorname{dim} C\right\rfloor\right\rceil+1 .
$$

Let $\mathcal{U}=\{V \subseteq \widehat{A}: V$ open and $\bar{V}$ compact $\}$, directed by inclusion. For $V \in \mathcal{U}$, $J_{V} \cap J_{(\widehat{A} \backslash \bar{V})}=\{0\}$ and so $J_{V}$ is isomorphic to a closed ideal of $A / J_{(\widehat{A} \backslash \bar{V})}$. Hence, by Lemma 1.1(a)(i),

$$
\operatorname{RR}\left(J_{V}\right) \leq \operatorname{RR}\left(A / J_{(\widehat{A} \backslash \bar{V})}=\left\lceil\frac{\operatorname{dim} \bar{V}}{2 n-1}\right\rceil .\right.
$$

Similarly, by Lemma 1.1(a)(ii),

$$
\operatorname{sr}\left(J_{V}\right) \leq \operatorname{sr}\left(A / J_{(\widehat{A} \backslash \bar{V})}=\left\lceil\frac{1}{n}\left\lfloor\frac{1}{2} \operatorname{dim} \bar{V}\right\rfloor\right\rceil+1 .\right.
$$

Since $\widehat{A}$ is locally compact, $\bigcup_{V \in \mathcal{U}} V=\widehat{A}$ and hence the ideal $\bigcup_{V \in \mathcal{U}} J_{V}$ is dense in $A$. Thus $A$ is the inductive limit of the directed family $\left\{J_{V}: V \in \mathcal{U}\right\}$ and so

$$
\operatorname{sr}(A) \leq \sup _{V \in \mathcal{U}} \operatorname{sr}\left(J_{V}\right) \text { and } \operatorname{RR}(A) \leq \sup _{V \in \mathcal{U}} \operatorname{RR}\left(J_{V}\right)
$$

(see [24, Theorem 5.1] and [14 Lemma 4(i)]). Combining these inequalities with (4), (5), (6) and (7), we obtain (i) and (ii).

(b) The proof is similar to (a), using Lemma 1.1(b) in place of Lemma 1.1(a). For this, note that if $V \in \mathcal{U}$, then $A / J_{(\widehat{A} \backslash \bar{V})}$ inherits all relevant properties from $A$, and its compact spectrum $\bar{V}$ satisfies $\operatorname{dim} \bar{V} \leq \operatorname{dim} \widehat{A}<\infty$.

Lemma 1.3. Let $A$ be a $C^{*}$-algebra and $n \geq 1$. Suppose that

$$
A=J_{0} \supseteq J_{1} \supseteq \ldots \supseteq J_{n}=\{0\}
$$

is a sequence of closed ideals of $A$ such that $J_{i} / J_{i+1}$ is homogeneous for $1 \leq i<n$. Then

(i) $\operatorname{sr}(A)=\max _{0 \leq i \leq n-1} \operatorname{sr}\left(J_{i} / J_{i+1}\right)$.

(ii) $\operatorname{RR}(A)=\max _{0 \leq i \leq n-1} \operatorname{RR}\left(J_{i} / J_{i+1}\right)$.

Proof. (i) This clearly holds for $n=1$. For $n>1$ we combine the fact that

$$
\operatorname{sr}(A)=\max \left\{\operatorname{sr}\left(J_{n-1}\right), \operatorname{sr}\left(A / J_{n-1}\right)\right\}
$$


[21, Lemma 5.a)] with the inductive assumption that

$$
\begin{aligned}
\operatorname{sr}\left(A / J_{n-1}\right) & =\max _{0 \leq i \leq n-2}\left\{\operatorname{sr}\left(\left(J_{i} / J_{n-1}\right) /\left(J_{i+1} / J_{n-1}\right)\right)\right\} \\
& =\max _{0 \leq i \leq n-2}\left\{\operatorname{sr}\left(J_{i} / J_{i+1}\right)\right\} .
\end{aligned}
$$

(ii) We note first of all that if a homogeneous $C^{*}$-algebra $J$ is a closed ideal of a $C^{*}$-algebra $B$, then

$$
\mathrm{RR}(B)=\max \{\operatorname{RR}(J), \operatorname{RR}(B / J)\} .
$$

The proof of this proceeds as in the case of stable rank [21 Lemma 5.a)], but uses the fact that the real rank does not increase on passing to a closed ideal and replaces the application of [26, Proposition 3.15] by [22] Lemma 1.9]. (Note that in the proof of [22, Lemma 1.9] there is sufficient surjectivity for the argument of [22, Proposition 1.3] to be satisfactorily applied. A full proof of [22 Proposition 1.3] has been given in [19, Proposition 1.6].) The proof of (ii) may now be completed as in (i), replacing sr by RR.

The next lemma will be needed for dealing with the $C^{*}$-algebras of Moore groups.

Lemma 1.4. Suppose that a $C^{*}$-algebra $A$ is the inductive limit of a directed system $\left(A_{\lambda}\right)_{\lambda \in \Lambda}$ of subhomogeneous closed ideals. For each $d \geq 0$, let $I_{d}$ be the closed ideal of $A$ such that

$$
\widehat{A / I_{d}}=\{\pi \in \widehat{A}: \operatorname{dim} \pi \leq d\} .
$$

Then, taking suprema in $\mathbb{N} \cup\{\infty\}$, we have

(i) $\operatorname{RR}(A)=\sup _{d \geq 0} \operatorname{RR}\left(I_{d} / I_{d+1}\right)$.

(ii) $\operatorname{sr}(A)=\sup _{d \geq 0} \operatorname{sr}\left(I_{d} / I_{d+1}\right)$.

(iii) $\operatorname{RR}(A) \leq \operatorname{dim} \widehat{A}$.

Proof. Temporarily fix $\lambda \in \Lambda$ and let $n$ be the largest dimension of an irreducible representation of $A_{\lambda}$. Let $q$ be the canonical quotient map $A \rightarrow A / I_{n}$. Then the restriction of $q$ to $A_{\lambda}$ is injective and $q\left(A_{\lambda}\right)$ is a closed ideal of $A / I_{n}$. Applying Lemma 1.3(ii) to $A / I_{n}$, we obtain that

$$
\mathrm{RR}\left(A_{\lambda}\right)=\operatorname{RR}\left(q\left(A_{\lambda}\right)\right) \leq \mathrm{RR}\left(A / I_{n}\right) \leq \max _{0 \leq d \leq n-1} \operatorname{RR}\left(I_{d} / I_{d+1}\right) .
$$

Hence

$$
\mathrm{RR}(A) \leq \sup _{\lambda} \mathrm{RR}\left(A_{\lambda}\right) \leq \sup _{d \geq 0} \operatorname{RR}\left(I_{d} / I_{d+1}\right),
$$

and (ii) is proved in a similar fashion.

If $C$ is a compact subset of $\widehat{I_{d} / I_{d+1}}$ for some $d \geq 0$, then it follows from [9], Corollary 1 of Section 10] that $C$ is closed in $\widehat{A}$. Thus $\operatorname{dim} C \leq \operatorname{dim} \widehat{A}$ and so $\operatorname{RR}\left(I_{d} / I_{d+1}\right) \leq \operatorname{dim} \widehat{A}$ by Proposition 1.2(a)(i). Hence (iii) follows from (i).

As a special case of Lemma 1.4(iii), we see that if $A$ is a subhomogeneous $C^{*}$ algebra, then $\operatorname{RR}(A) \leq \operatorname{dim} \widehat{A}$ (this is stated in [22] Corollary 1.8], but the proof there uses an open cover of a kind which need not exist). As might be expected from the proof above, this inequality may be very far from sharp. For example, let $A$ be the $C^{*}$-subalgebra of the algebra $C\left([0,1], M_{n}(\mathbb{C})\right)$ consisting of those functions $f$ for which $f(1)$ is a diagonal matrix. Applying Lemma 1.3 and Proposition 1.2(a)(i) to the composition series $A \supseteq\{f \in A: f(1)=0\} \supseteq\{0\}$, one easily sees that $\operatorname{RR}(A)=1$. However, $\widehat{A}$ is homeomorphic to $[0,1)$ with $n$ "points at infinity" 
replacing 1. A simple application of [23, Chapter 3, Proposition 1.2, (a) $\Leftrightarrow$ (b)] shows that $\operatorname{dim} \widehat{A} \geq n-1$, and a more elaborate application of the same proposition of [23] shows that in fact $\operatorname{dim} \widehat{A}=n-1$. The same phenomenon occurs within the context of group $C^{*}$-algebras (see Section 4 ).

\section{Groups With Relatively COMPaCt CONJugacy Classes}

Let $X$ be a non-compact locally compact Hausdorff space and let $X^{+}$be the one-point compactification of $X$. Then, by 223 . Chapter 3, Proposition 1.5 and Proposition 5.6],

$$
\operatorname{dim} X^{+}=\sup \{\operatorname{dim} C: C \subseteq X, C \text { compact }\} \leq \operatorname{dim} X .
$$

However, if $X$ is a locally compact group, then $X$ is paracompact and normal [12. Theorem 8.13] and hence $\operatorname{dim} X=\operatorname{dim} X^{+}$by [23, Chapter 3, Proposition 6.9].

Let $G$ be a locally compact group. An element $x$ of $G$ is called compact if the closed subgroup generated by $x$ is compact. Let $G^{c}$ denote the set of all compact elements of $G$. When $G$ is discrete, $G^{c}$ is just the set of elements of finite order which is then usually denoted $G^{t}$ and called the set of torsion elements of $G$.

In general, $G^{c}$ is neither a subgroup of $G$ nor closed in $G$. However, $G^{c}$ is a closed (normal) subgroup of $G$ whenever $G$ is a group with relatively compact conjugacy classes (a so-called $[\mathrm{FC}]^{-}$group). For example, every locally compact group whose commutator subgroup is compact is an $[\mathrm{FC}]^{-}$group. The structure theory of $[\mathrm{FC}]^{-}$groups has been developed in 11]. For such $G$, we shall exploit the following properties:

(1) $G / G^{c}$ is compact-free (i.e., $\left(G / G^{c}\right)^{c}=\left\{G^{c}\right\}$ [11, Theorem 3.16]).

(2) $G / G^{c}$ is abelian and hence the direct product of a vector group $V=\mathbb{R}^{k}$ and a discrete torsion-free abelian group $D$ [11, Theorem 3.16].

(3) If $G$ is compactly generated, then $G^{c}$ is compact [11, Theorem 3.20]. We now define the rank of $G, \operatorname{rank} G$, to be the rank of the abelian locally compact group $G / G^{c}$, that is, $\operatorname{rank} G=\operatorname{rank}\left(G / G^{c}\right)=k+\operatorname{rank} D$, where for a discrete abelian group $D, \operatorname{rank} D$ means the torsion-free rank (see [12, Appendix A]). It is then clear that $\operatorname{rank} G=\operatorname{rank}(G / K)$ for every compact normal subgroup $K$ of $G$.

The purpose of this section is to investigate $\operatorname{RR}\left(C^{*}(G)\right)$ and $\operatorname{sr}\left(C^{*}(G)\right)$ for $[\mathrm{FC}]^{-}$ groups $G$. Recall that if $G$ has the property that every compact subset of $G$ generates a compact subgroup, then $C^{*}(G)$ is the inductive limit of finite-dimensional subalgebras and hence $\operatorname{sr}\left(C^{*}(G)\right)=1$ and $\operatorname{RR}\left(C^{*}(G)\right)=0$ [24, Proposition 3.5; 14, Lemma 4]. This leads to the expectation that the ranks of $C^{*}(G)$ might depend only on the structure of the quotient group $G / G^{c}$. In fact, it turns out that these ranks are closely related to $\operatorname{rank} G=\operatorname{rank}\left(G / G^{c}\right)$.

To start with, we consider abelian locally compact groups $G$. Then $C^{*}(G)=$ $C_{0}(\widehat{G})$ and hence, using the results of the opening paragraph,

$$
\operatorname{RR}\left(C^{*}(G)\right)=\operatorname{dim} \widehat{G} \text { and } \operatorname{sr}\left(C^{*}(G)\right)=\left\lfloor\frac{1}{2} \operatorname{dim} \widehat{G}\right\rfloor+1 .
$$

On the other hand, $\operatorname{dim} \widehat{G}=\operatorname{rank} G$. To see this, note that by the structure theory of locally compact abelian groups, $G=\mathbb{R}^{k} \times H$, where $H$ contains a compact subgroup $K$ which is open in $H$. Then $\widehat{G}$ is the disjoint union of cosets $\chi \cdot \widehat{G / K}, \chi \in \widehat{G}$, and each such coset is open (and hence closed) in $\widehat{G}$ and homeomorphic to $\widehat{G / K}$. Thus $\operatorname{dim} \widehat{G}=\operatorname{dim} \widehat{G / K}$. Therefore, in order to verify that $\operatorname{dim} \widehat{G}=\operatorname{rank} G$, we can 
assume that $G=\mathbb{R}^{k} \times D$ where $D$ is discrete. Then $\widehat{G}=\mathbb{R}^{k} \times \widehat{D}$ and, by [12] Theorem 24.28], $\operatorname{dim} \widehat{D}=\operatorname{rank} D$. Now, for topological spaces $X$ and $Y$, only in very specific cases does the product theorem $\operatorname{dim}(X \times Y)=\operatorname{dim} X+\operatorname{dim} Y$ hold, for example if $X$ is a compact interval and $Y$ is a normal space (see [17, p.152 and Section 42]). With $B_{m}=[-m, m]^{k} \subseteq \mathbb{R}^{k}$ for $m \geq 1$, it follows by the countable sum theorem for the (normal) group $\mathbb{R}^{k} \times \widehat{D}$ that

$$
\operatorname{dim}\left(\mathbb{R}^{k} \times \widehat{D}\right)=\sup _{m}\left\{\operatorname{dim}\left(B_{m} \times \widehat{D}\right)\right\}=k+\operatorname{dim} \widehat{D}
$$

as was to be shown.

Lemma 2.1. Let $G$ be a locally compact group with a compact normal subgroup $K$ such that $G / K$ is abelian, and suppose that $G$ is type I. Then $\operatorname{dim} \widehat{G}=\operatorname{dim}(\widehat{G / K})$.

Proof. For $\pi \in \widehat{G}$, let

$$
\pi \otimes \widehat{G / K}=\{\pi \otimes \lambda: \lambda \in \widehat{G / K}\} .
$$

Then $\widehat{G}$ can be written as a disjoint union of such 'cosets' $\pi \otimes \widehat{G / K}, \pi \in \widehat{G}$, each $\pi \otimes \widehat{G / K}$ is open in $\widehat{G}$, and the mapping $\lambda \rightarrow \pi \otimes \lambda$ from $\widehat{G / K}$ onto $\pi \otimes \widehat{G / K}$ is continuous and open [13, Theorem 2 and Proposition]. The latter fact implies that $\pi \otimes \widehat{G / K}$ is homeomorphic to $\widehat{H_{\pi} / K}$, where $H_{\pi}$ is the closed subgroup of $G$ defined by

It follows that

$$
\widehat{G / H_{\pi}}=\{\chi \in \widehat{G / K}: \pi \otimes \chi=\pi\} .
$$

$$
\operatorname{dim}(\pi \otimes \widehat{G / K})=\operatorname{dim} \widehat{H_{\pi} / K}=\operatorname{rank}\left(H_{\pi} / K\right) \leq \operatorname{rank}(G / K)=\operatorname{dim}(\widehat{G / K})
$$

for every $\pi \in \widehat{G}$. Since the sets $\pi \otimes \widehat{G / K}$ are open and closed in $\widehat{G}$, we conclude that $\operatorname{dim} \widehat{G}=\operatorname{dim}(\widehat{G / K})$.

Lemma 2.2. Let $K$ be a compact normal subgroup of the locally compact group $G$ and let $\Sigma$ be a representative system of the $G$-orbits in $\widehat{K}$. For $\sigma \in \Sigma$, let $\pi_{\sigma}=\operatorname{ind}_{K}^{G} \sigma$ and $C_{\pi_{\sigma}}^{*}(G)=\pi_{\sigma}\left(C^{*}(G)\right)$. Then

$$
\operatorname{sr}\left(C_{r}^{*}(G)\right)=\sup _{\sigma \in \Sigma} \operatorname{sr}\left(C_{\pi_{\sigma}}^{*}(G)\right)
$$

and

$$
\operatorname{RR}\left(C_{r}^{*}(G)\right)=\sup _{\sigma \in \Sigma} \operatorname{RR}\left(C_{\pi_{\sigma}}^{*}(G)\right) .
$$

Proof. Let $A$ denote the $c_{0}$-direct sum of $C^{*}$-algebras $C_{\pi_{\sigma}}^{*}(G), \sigma \in \Sigma$. We claim that $C_{r}^{*}(G)$ is isomorphic to $A$. Once this has been shown, the two statements follow from [24, Theorem 5.2] and [14, Lemma 4].

Since $\bigcup_{\sigma \in \Sigma} G(\sigma)=\widehat{K}$, the set of representations $\pi_{\sigma}, \sigma \in \Sigma$, is weakly equivalent to the regular representation. Thus $\bigcup_{\sigma \in \Sigma} \operatorname{supp} \pi_{\sigma}$ is dense in $\widehat{G}_{r}$. In fact, these two sets are equal. To see this, let $\tau \in \widehat{G}_{r}$ and $\sigma \in \widehat{K}$ such that $\tau \mid K \sim G(\sigma)$. There exists a net $\left(\tau_{\alpha}\right)_{\alpha}$ such that $\tau_{\alpha} \rightarrow \tau$ in $\widehat{G}_{r}$ and $\tau_{\alpha} \in \operatorname{supp} \pi_{\sigma_{\alpha}}$ for some $\sigma_{\alpha} \in \Sigma$. Since $\tau_{\alpha}|K \rightarrow \tau| K$ and $\widehat{K}$ is discrete, we can assume that $\sigma_{\alpha}=\sigma$ for all $\alpha$. Thus $\tau_{\alpha} \in \operatorname{supp} \pi_{\sigma}$ for all $\alpha$, whence $\tau \in \operatorname{supp} \pi_{\sigma}$. Notice also that, since $\widehat{K}$ is discrete, the sets $\operatorname{supp} \pi_{\sigma}, \sigma \in \Sigma$, are open in $\widehat{G}_{r}$. 
Now, given $f \in C_{r}^{*}(G)$ and $\epsilon>0$, by the Riemann-Lebesgue Lemma [3 (3.3.7)], there exists a compact subset $C$ of $\widehat{G}_{r}$ such that $\|\tau(f)\| \leq \epsilon$ for all $\tau \in \widehat{G}_{r} \backslash C$. Since the sets supp $\pi_{\sigma}$ are open in $\widehat{G}_{r}$ and cover $\widehat{G}_{r}, C \subseteq \bigcup_{j=1}^{n} \operatorname{supp} \pi_{\sigma_{j}}$ for certain $\sigma_{1}, \ldots, \sigma_{n} \in \Sigma$. It follows that $\left\|\pi_{\sigma}(f)\right\| \leq \epsilon$ for $\sigma \in \Sigma, \sigma \neq \sigma_{j}, 1 \leq j \leq n$. So the function $\sigma \rightarrow\left\|\pi_{\sigma}(f)\right\|$ on $\Sigma$ vanishes at infinity. We conclude that $f \rightarrow\left(\pi_{\sigma}(f)\right)_{\sigma \in \Sigma}$ is an isometric isomorphism from $C_{r}^{*}(G)$ into $A$. To prove that this map is onto, it remains to show that, given any $f \in C_{r}^{*}(G)$ and $\sigma_{0} \in \Sigma$, there exists $g \in C_{r}^{*}(G)$ such that $\pi_{\sigma_{0}}(g)=\pi_{\sigma_{0}}(f)$ and $\pi_{\sigma}(g)=0$ for all $\sigma \neq \sigma_{0}$. To that end, consider the closed ideals

$$
I=\bigcap\left\{\operatorname{ker} \pi_{\sigma}: \sigma \in \Sigma, \sigma \neq \sigma_{0}\right\} \text { and } J=\operatorname{ker} \pi_{\sigma_{0}}
$$

of $C_{r}^{*}(G)$. Then $I+J=C_{r}^{*}(G)$ and hence $f=g+h$ with $g \in I$ and $h \in J$. It follows that $\pi_{\sigma}(g)=0$ for all $\sigma \neq \sigma_{0}$ and $\pi_{\sigma_{0}}(g)=\pi_{\sigma_{0}}(f)$, as required.

For the proof of the next lemma we need the following technical preparation.

Remark 2.3. Let $K$ be a compact normal subgroup of $G$ such that $G / K$ is second countable, and let $\sigma$ be an irreducible representation of $K$ and $\pi=\operatorname{ind}_{K}^{G} \sigma$. Then the $C^{*}$-algebra $\pi\left(C^{*}(G)\right)$ is separable. For that, it suffices to show that there is a closed normal subgroup $N$ of $G$ such that $G / N$ is second countable and $\pi(N)=\left\{1_{\pi}\right\}$. Let

$$
N=\left\{y \in K: \sigma\left(x^{-1} y x\right)=1_{\sigma} \text { for all } x \in G\right\} .
$$

Then $N$ is normal in $G$ and $\pi(N)=\left\{1_{\pi}\right\}$, since $\pi_{\mid K} \sim G(\sigma)$. Since $G / K$ is second countable, it is enough to show that $K / N$ is second countable. Now, $K$ being compact, we only need to verify that $\{N\}$ has a countable neighbourhood basis in $K / N$. To see this, notice that since $\widehat{K}$ is discrete, the stability group $G_{\sigma}$ of $\sigma$ is open in $G$. As $G / K$ is second countable, it follows that $G / G_{\sigma}$ is countable and hence so is $G(\sigma) \subseteq \widehat{K}$. Therefore, $N$ is the countable intersection of closed normal subgroups of $K$ with Lie quotients. This implies that $\{N\}$ has a countable neighbourhood basis in $K / N$.

Lemma 2.4. Suppose that $G$ is a type I group and contains a compact normal subgroup $K$ such that $G / K=\mathbb{R}^{k} \times \mathbb{Z}^{l}\left(k, l \in \mathbb{N}_{0}\right)$. Then

$$
\operatorname{sr}\left(C^{*}(G)\right)=\left\lfloor\frac{k+l}{2}\right\rfloor+1 \text { and } \operatorname{RR}\left(C^{*}(G)\right)=k+l .
$$

Proof. Recall first that, since $C^{*}(G / K)$ is a quotient of $C^{*}(G)$ and $C^{*}(G / K)=$ $C_{0}(\widehat{G / K})=C_{0}\left(\mathbb{R}^{k} \times \mathbb{T}^{l}\right)$, we have

$$
\operatorname{sr}\left(C^{*}(G)\right) \geq \operatorname{sr}\left(C^{*}(G / K)\right)=\left\lfloor\frac{k+l}{2}\right\rfloor+1
$$

and

$$
\operatorname{RR}\left(C^{*}(G)\right) \geq \operatorname{RR}\left(C^{*}(G / K)\right)=k+l .
$$

Thus, retaining the notation of Lemma 2.2 , since $G$ is amenable it suffices to show that

$$
\operatorname{sr}\left(C_{\pi_{\sigma}}^{*}(G)\right) \leq\left\lfloor\frac{k+l}{2}\right\rfloor+1 \text { and } \operatorname{RR}\left(C_{\pi_{\sigma}}^{*}(G)\right) \leq k+l
$$

for each $\sigma \in \widehat{K}$. Fix $\sigma$ and choose $\tau \in \widehat{G}$ such that $\tau \mid K \geq \sigma$. Then, since $G$ is type I, $\widehat{C_{\pi_{\sigma}}^{*}(G)}=\tau \otimes \widehat{G / K}$. Retaining the notation of the proof of Lemma 2.1, we 
have a homeomorphism between $\widehat{H_{\tau} / K}$ and $\tau \otimes \widehat{G / K}$. Clearly, $H_{\tau} / K=\mathbb{R}^{m} \times \mathbb{Z}^{n}$ with $m+n \leq k+l$. Thus $\operatorname{dim}(\tau \otimes \widehat{G / K}) \leq k+l$. Moreover $C^{*}(G)$ is a continuous trace algebra (see 7, Lemma 6]). Now, let $\tau$ be of finite dimension $n$. Then, by Proposition 1.2(a)(ii),

$$
\operatorname{sr}\left(C_{\pi_{\sigma}}^{*}(G)\right) \leq\left\lceil\frac{1}{n}\left\lfloor\frac{1}{2} \operatorname{dim} \widehat{C_{\pi_{\sigma}}^{*}(G)}\right\rfloor\right\rceil+1 \leq\left\lceil\frac{1}{n}\left\lfloor\frac{k+l}{2}\right\rfloor\right\rceil+1 \leq\left\lfloor\frac{k+l}{2}\right\rfloor+1
$$

and, by Proposition 1.2(a)(i),

$$
\operatorname{RR}\left(C_{\pi_{\sigma}}^{*}(G)\right) \leq\left\lceil\frac{\operatorname{dim} \widehat{C_{\pi_{\sigma}}^{*}(G)}}{2 n-1}\right\rceil \leq\left\lceil\frac{k+l}{2 n-1}\right\rceil \leq k+l .
$$

Finally, suppose that $\tau$ is infinite dimensional. Since $C_{\pi_{\sigma}}^{*}(G)$ is separable (Remark 2.3), $\operatorname{dim} \omega=\aleph_{0}$ for every $\omega \in \widehat{C_{\pi_{\sigma}}^{*}(G)}=\tau \otimes \widehat{G / K}$. Since $\operatorname{dim} \widehat{C_{\pi_{\sigma}}^{*}(G)} \leq k+l$, Proposition 1.2(b) yields that

and

$$
\operatorname{sr}\left(C_{\pi_{\sigma}}^{*}(G)\right) \leq \min \left\{2,1+\left\lfloor\frac{1}{2} \operatorname{dim} \widehat{C_{\pi_{\sigma}}^{*}(G)}\right\rfloor\right\} \leq 1+\left\lfloor\frac{k+l}{2}\right\rfloor
$$

$$
\operatorname{RR}\left(C_{\pi_{\sigma}}^{*}(G)\right) \leq \min \left\{1, \operatorname{dim} \widehat{C_{\pi_{\sigma}}^{*}(G)}\right\} \leq k+l .
$$

Theorem 2.5. Let $G$ be a locally compact group with relatively compact conjugacy classes. Then the following conditions are equivalent:

(i) $\operatorname{sr}\left(C^{*}(G)\right)<\infty$.

(ii) $\operatorname{RR}\left(C^{*}(G)\right)<\infty$.

(iii) The abelian group $G / G^{c}$ has finite rank.

Proof. (i) $\Rightarrow$ (ii) follows from the fact that, for any $C^{*}$-algebra $A, \operatorname{RR}(A) \leq 2 \operatorname{sr}(A)-$ 1 [2] Proposition 2.1].

To show (ii) $\Rightarrow$ (iii), let $H=G / G^{c}$. Since $C^{*}(H)=C_{0}(\widehat{H})$ is a quotient of $C^{*}(G)$, we have

$$
\operatorname{RR}\left(C^{*}(G)\right) \geq \operatorname{RR}\left(C_{0}(\widehat{H})\right)=\operatorname{dim} \widehat{H}=\operatorname{rank} H .
$$

Finally, suppose that $n=\operatorname{rank}\left(G / G^{c}\right)<\infty$. By the structure theory of compactfree locally compact abelian groups, $G / G^{c}=\mathbb{R}^{k} \times D$, where $k \leq n, D$ is discrete and $D$ contains a free abelian group $F$ of rank $n-k$ such that $D / F$ is a torsion group. In particular, each finitely generated subgroup of $D$ containing $F$ is isomorphic to $\mathbb{Z}^{n-k}$ because it is torsion-free. Let $C$ be a compact subset of $G$ such that $C \cup G^{c}$ generates the inverse image of $\mathbb{R}^{k} \times F$ in $G$. Let $\mathcal{H}$ denote the collection of all compactly generated open subgroups of $G$ containing $C$. Then, since $C^{*}(G)$ is the inductive limit of $C^{*}$-subalgebras $C^{*}(H), H \in \mathcal{H}$,

$$
\operatorname{sr}\left(C^{*}(G)\right) \leq \sup _{H \in \mathcal{H}} \operatorname{sr}\left(C^{*}(H)\right) .
$$

Thus, to establish (i), it suffices to show that

$$
\operatorname{sr}\left(C^{*}(H)\right) \leq\lfloor k / 2\rfloor+n-k+1
$$

for each $H \in \mathcal{H}$. Now, for $H \in \mathcal{H}, H^{c}$ is compact since $H$ is compactly generated and

$$
H / H^{c}=H / H \cap G^{c}=H G^{c} / G^{c} \supseteq \mathbb{R}^{k} \times F,
$$


and $\mathbb{R}^{k} \times F$ has finite index in $H / H^{c}$. Hence $H / H^{c}$, being compact-free, is isomorphic to $\mathbb{R}^{k} \times \mathbb{Z}^{n-k}$. Let $K$ be the inverse image of $\mathbb{R}^{k}$ in $H$. Then $K$ is of type I and hence $\operatorname{sr}\left(C^{*}(K)\right)=\lfloor k / 2\rfloor+1$ (Lemma 2.4). Since, by [24, Theorem 7.1], for an arbitrary $C^{*}$-algebra $A$ and any action $\alpha$ of $\mathbb{Z}$ on $A, \operatorname{sr}\left(A \times_{\alpha} \mathbb{Z}\right) \leq \operatorname{sr}(A)+1$, it follows easily by induction that

$$
\operatorname{sr}\left(C^{*}(H)\right) \leq \operatorname{sr}\left(C^{*}(K)\right)+n-k=\lfloor k / 2\rfloor+n-k+1 .
$$

This finishes the proof of (iii) $\Rightarrow$ (i).

Theorem 2.6. Let $G$ be a locally compact group with relatively compact conjugacy classes, and suppose that $G / G^{c}$ has finite rank $n$ and that every compactly generated open subgroup of $G$ is of type $I$. Then

$$
\operatorname{sr}\left(C^{*}(G)\right)=\left\lfloor\frac{n}{2}\right\rfloor+1 \text { and } \operatorname{RR}\left(C^{*}(G)\right)=n .
$$

Proof. Let $K=G / G^{c}$. Then (compare the proof of Theorem 2.5) we have $\operatorname{RR}\left(C^{*}(G)\right) \geq \operatorname{RR}\left(C^{*}(K)\right)=n$ and

$$
\operatorname{sr}\left(C^{*}(G)\right) \geq \operatorname{sr}\left(C^{*}(K)\right)=\operatorname{sr}\left(C_{0}(\widehat{K})\right)=\left\lfloor\frac{1}{2} \operatorname{dim} \widehat{K}\right\rfloor+1=\lfloor n / 2\rfloor+1 .
$$

Now, let $\mathcal{H}$ be as in the proof of Theorem 2.5. Since every $H \in \mathcal{H}$ is of type I and $H / H^{c}$ is isomorphic to $\mathbb{R}^{k} \times \mathbb{Z}^{l}$, with $k+l=n$, we conclude from Lemma 2.4 that

$$
\operatorname{sr}\left(C^{*}(H)\right)=\left\lfloor\frac{n}{2}\right\rfloor+1 \text { and } \operatorname{RR}\left(C^{*}(H)\right)=n .
$$

This implies that $\operatorname{sr}\left(C^{*}(G)\right) \leq\lfloor n / 2\rfloor+1$ and $\operatorname{RR}\left(C^{*}(G)\right) \leq n$.

Since every finitely generated $F C$-group has a centre of finite index and hence is type I, the following corollary is an immediate consequence of Theorems 2.5 and 2.6 .

Corollary 2.7. Let $G$ be a discrete $F C$-group and let $G^{t}$ be the torsion subgroup of $G$.

(i) If $G / G^{t}$ is of finite rank l, then

$$
\operatorname{sr}\left(C^{*}(G)\right)=\left\lfloor\frac{l}{2}\right\rfloor+1 \text { and } \operatorname{RR}\left(C^{*}(G)\right)=l .
$$

(ii) If $G / G^{t}$ has infinite rank, then

$$
\operatorname{sr}\left(C^{*}(G)\right)=\operatorname{RR}\left(C^{*}(G)\right)=\infty .
$$

The following lemma will be used several times throughout the paper.

Lemma 2.8. Let $G$ be a locally compact group with relatively compact conjugacy classes and let $H$ be a closed subgroup of finite index in $G$. Then $\operatorname{rank} G=\operatorname{rank} H$.

Proof. Recall first that $G / G^{c}$ is the direct product of a vector group and a torsionfree discrete abelian group, and similarly for $H / H^{c}$. Define open subgroups $K$ and $L$ of $G$ by $G^{c} \subseteq K$ and $K / G^{c}=\left(G / G^{c}\right)_{0}$ and $H^{c} \subseteq L$ and $L / H^{c}=\left(H / H^{c}\right)_{0}$. Let $q: G \rightarrow G / G^{c}$ denote the quotient homomorphism. Then $q(L)$ is a connected open subgroup of $G / G^{c}$, whence $q(L)=K / G^{c}$. Since $L / H^{c}$ is $\sigma$-compact and $H^{c}=H \cap G^{c}$, it follows that $q$ induces a topological isomorphism between $L / H^{c}$ and $K / G^{c}$ (see [12, Theorem 5.29]). So $\left(H / H^{c}\right)_{0}$ and $\left(G / G^{c}\right)_{0}$ are isomorphic vector groups. Therefore it remains to prove that the torsion-free discrete abelian groups $H / L$ and $G / K$ have the same rank. Now, since $H / H^{c}=H / H \cap G^{c}$ is algebraically 
isomorphic to $H G^{c} / G^{c}$, which is a subgroup of finite index in $G / G^{c}$, we obtain that $H / L$ is isomorphic to a subgroup of finite index of $G / K$. Therefore we only need to observe that if $A$ is a torsion-free abelian group and $B$ is a subgroup of finite index in $A$, then $\operatorname{rank} A=\operatorname{rank} B$. This, however, follows from the very definition of rank [12, Appendix A]. Indeed, given $x_{1}, \ldots, x_{r} \in A$ there exists $m \in \mathbb{N}$ such that $x_{j}^{m} \in B(1 \leq j \leq r)$. Then, for $n_{1}, \ldots, n_{r} \in \mathbb{Z},\left(x_{1}^{m}\right)^{n_{1}} \cdot \ldots \cdot\left(x_{1}^{m}\right)^{n_{r}}=e$ if and only if $x_{1}^{n_{1}} \cdot \ldots \cdot x_{1}^{n_{r}}=e$.

Corollary 2.9. Let $G$ be a locally compact group with relatively compact conjugacy classes and suppose that every compactly generated open subgroup of $G$ is type $I$. Let $H$ be a closed subgroup of finite index in $G$. Then

$$
\operatorname{sr}\left(C^{*}(G)\right)=\operatorname{sr}\left(C^{*}(H)\right)
$$

and

$$
\operatorname{RR}\left(C^{*}(G)\right)=\operatorname{RR}\left(C^{*}(H)\right)
$$

Proof. The statement follows immediately from Theorems 2.5 and 2.6 and Lemma 2.8 .

\section{Groups With Finite-Dimensional IRREDUCiBle REPRESENTATIONS: UPPER AND LOWER BOUNDS FOR THE RANKS}

In this section we concentrate on locally compact groups all of whose irreducible representations are finite dimensional. Such groups have been completely characterized by Moore [16] and are therefore usually referred to as Moore groups. The class of Moore groups includes all central groups, where a locally compact group $G$ is called central if its centre $Z(G)$ is cocompact in $G$. By [16, Theorem 2] a Lie group is a Moore group if and only if it contains a subgroup of finite index which is central. Moreover, every Moore group is a projective limit of Lie groups. Consequently, we have in particular the following structural properties. Let $G_{F}$ denote the subgroup of $G$ consisting of all elements with relatively compact conjugacy classes. Then $G_{F}$ is an open subgroup of finite index in $G$, and the commutator subgroup $\left[G_{F}, G_{F}\right]$ of $G_{F}$ is compact.

A locally compact group $G$ is said to be of bounded representation dimension if there exists some $N \in \mathbb{N}$ such that $\operatorname{dim} \pi \leq N$ for all irreducible representations $\pi$ of $G$. By [16, Theorem 1] this condition is equivalent to $G$ containing an abelian closed normal subgroup of finite index.

For a $C^{*}$-algebra $A$ and $d \in \mathbb{N}$, let $\widehat{A}_{d}=\{\pi \in \widehat{A}: \operatorname{dim} \pi=d\}$. By [4, Proposition 3.6.3] $\widehat{A}_{d}$ is open in $\bigcup_{n=1}^{d} \widehat{A}_{n}$. If $G$ is a locally compact group, let

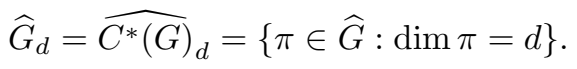

Our purpose is to establish, for Moore groups $G$, upper and lower bounds for the ranks of $C^{*}(G)$.

Remark. In the proof of the next lemma we shall use the fact that if $H$ is a locally compact group and $C$ is any compact subset of $H$ with non-empty interior, then $\operatorname{dim} H=\operatorname{dim} C$. To see this, let $L$ denote the subgroup of $H$ generated by $C$. Then $L$ is open and, since $L$ is $\sigma$-compact, $L$ is a countable union of sets $x C, x \in L$. Since $L$ is a normal space [12, Theorem 8.13], the countable sum theorem yields that $\operatorname{dim} L=\operatorname{dim} C$. On the other hand, $\operatorname{dim} H=\operatorname{dim} L$, since the cosets of $L$ are open and closed. 
Lemma 3.1. Let $G$ be a Moore group and let $K=\left[G_{F}, G_{F}\right]$. Then there exists a closed ideal $J$ of $C^{*}(G)$ with the following properties:

(i) $C^{*}(G) / J$ is $\left[G: G_{F}\right]$-homogeneous.

(ii) $\left(C^{*}(G) / J\right)^{\wedge}$ is compact and $\operatorname{dim}\left(\left(C^{*}(G) / J\right)^{\wedge}\right)=\operatorname{rank}\left(G_{F} / K\right)$.

Proof. For $\chi \in \widehat{G_{F} / K}$, let $G_{\chi}$ be the stabiliser of $\chi$ in $G$. Let $V=\left\{\chi \in \widehat{G_{F} / K}\right.$ : $\left.G_{\chi}=G_{F}\right\}$. Then $V$ is open and dense in $\widehat{G_{F} / K}$ [15. Lemma 2]. Since the $G$ orbits in $\widehat{G_{F} / K}$ are finite, we can choose a compact subset $C$ of $V$ such that $\stackrel{\circ}{C} \neq \emptyset$ and $C$ meets every $G$-orbit in $\widehat{G_{F} / K}$ in at most one point. Then the mapping $i: \chi \rightarrow \operatorname{ind}_{G_{F}}^{G} \chi$ is a homeomorphism between $C$ and the subset $i(C)$ of $\widehat{G}_{\left[G: G_{F}\right]}$. By [9, Corollary 1 of Section 10] the compact set $i(C)$ is closed in $\widehat{G}$. Thus $i(C)=$ $\left(C^{*}(G) / J\right)^{\wedge}$ for some closed ideal $J$ of $C^{*}(G)$ and, by the above remark, $\operatorname{dim} i(C)=$ $\operatorname{dim} C=\operatorname{dim}\left(\widehat{G_{F} / K}\right)=\operatorname{rank}\left(G_{F} / K\right)$.

Lemma 3.2. Let $L$ be a Moore group and let $M$ be a closed normal subgroup of $L$ such that $M$ is a central group and $M$ has finite index in $L$. Let

$$
\widehat{M}_{L}=\left\{\tau \in \widehat{M}: \tau^{x}=\tau \text { for all } x \in L\right\}
$$

and

$$
\widehat{L}^{M}=\left\{\sigma \in \widehat{L}: \sigma \mid M \text { is a multiple of } \tau \text { for some } \tau \in \widehat{M}_{L}\right\} .
$$

Then $\operatorname{dim} D \leq \operatorname{rank} M$ for every closed subset $D$ of $\widehat{L}^{M}$.

Proof. Define a closed subgroup $K$ of $M$ by

$$
K=\left\{x \in M: \tau(x)=1_{\tau} \text { for all } \tau \in \widehat{M}_{L}\right\} .
$$

Since the elements of $\widehat{M}_{L}$ are $L$-invariant, $K$ is normal in $L$. Moreover, $\widehat{M}_{L}$ separates the cosets of $K$ in $M$. Thus, after passing to $L / K$ we can assume that $\widehat{M}_{L}$ separates the points of $M$.

Let $Z$ denote the centre of $M$. Then, for all $\tau \in \widehat{M}_{L}, \tau \mid Z$ is a multiple of a character $\chi_{\tau}$ of $Z$ and $\chi_{\tau}$ is $L$-invariant. Since $\widehat{M}_{L}$ separates the points of $M$, the set $\left\{\chi_{\tau}: \tau \in \widehat{M}_{L}\right\}$ separates the points of $Z$. It follows that $Z$ is contained in the centre of $L$. Since $L / Z$ is compact, the commutator subgroup $[L, L]$ of $L$ is compact. Using Lemma 2.1, we conclude that

$$
\begin{aligned}
\operatorname{dim} D & \leq \operatorname{dim} \widehat{L}=\operatorname{dim}(\widehat{L /[L, L}])=\operatorname{rank}(L /[L, L]) \\
& =\operatorname{rank}(M[L, L] /[L, L])=\operatorname{rank}(M / M \cap[L, L])=\operatorname{rank} M,
\end{aligned}
$$

as was to be shown.

Proposition 3.3. Let $G$ be a Moore group and let $C$ be a compact subset of $\widehat{G}_{d}$ for some $d \in \mathbb{N}$. Then $\operatorname{dim} C \leq \operatorname{rank}\left(G_{F}\right)$.

Proof. Recall first that $G$ is a projective limit of Lie groups $G / K_{\alpha}$ 16, Lemmas 4.2 and 4.3]. Then, by [16] Proposition 2.2], $\widehat{G}=\bigcup_{\alpha} \widehat{G / K_{\alpha}}$. Since $\widehat{G / K_{\alpha}}$ is open in $\widehat{G}$ and $C$ is compact, we have that $C \subseteq \widehat{G / K_{\alpha}}$ for some $\alpha$. Thus, after passing to $G / K_{\alpha}$, we can assume that $G$ is a Lie group. Then, by Theorem 2 of [16], $G$ has a subgroup of finite index which is central. Now, it is not difficult to verify that an open subgroup of a central group is again central. Therefore there exists a closed normal subgroup $N$ of finite index in $G$ that is central. Notice that $\operatorname{rank} N=\operatorname{rank}\left(G_{F}\right)$ since $N$ has finite index in $G_{F}$. 
The proof that follows turns out to be fairly technical and we first have to introduce some notation. For $\tau \in \widehat{N}$, let

$$
G_{\tau}=\left\{x \in G: \tau^{x}=\tau\right\},
$$

the stabiliser of $\tau$ in $G$. Let $\mathcal{S}$ be the collection of all stability groups $G_{\tau}, \tau \in \widehat{N}$. Since $N$ has only finite-dimensional irreducible representations and $\widehat{N}$ is a Hausdorff space, Mackey's theory provides a simple description of $\widehat{G}$ as follows.

Given $\pi \in \widehat{G}$, there exist $\tau \in \widehat{N}$ and $\sigma \in \widehat{G_{\tau}}$ such that $\sigma \mid N$ is a multiple of $\tau$ and $\pi=\operatorname{ind}_{G_{\tau}}^{G} \sigma$. Conversely, if $\tau \in \widehat{N}$ and $\sigma \in \widehat{G_{\tau}}$ are such that $\sigma \mid N$ is a multiple of $\tau$, then $\operatorname{ind}_{G_{\tau}}^{G} \sigma$ is irreducible. For $S \in \mathcal{S}$, let

$$
\begin{gathered}
\widehat{N}_{S}=\left\{\tau \in \widehat{N}: G_{\tau}=S\right\}, \\
\widehat{S}_{S}=\left\{\sigma \in \widehat{S}: \sigma \mid N \text { is a multiple of } \tau \text { for some } \tau \in \widehat{N}_{S}\right\},
\end{gathered}
$$

and $\widehat{G}_{S}=\left\{\operatorname{ind}_{S}^{G} \sigma: \sigma \in \widehat{S}_{S}\right\}$. For each $S \in \mathcal{S}$, the map $i_{S}: \sigma \rightarrow \operatorname{ind}_{S}^{G} \sigma$ is a homeomorphism between $\widehat{S}_{S}$ and $\widehat{G}_{S}$. Concerning Mackey's theory we refer to [10].

Notice next that if $\pi \in \widehat{G}$ is in the closure of $\widehat{G}_{S}$, then $\pi \in \widehat{G}_{T}$ for some $T \in \mathcal{S}$ containing $S$. Indeed, let $\left(\pi_{\alpha}\right)_{\alpha}$ be a net in $\widehat{G}_{S}$ converging to $\pi$ and let $\tau_{\alpha} \in \widehat{N}_{S}$ such that $\pi_{\alpha} \mid N \sim G\left(\tau_{\alpha}\right)$. Then $\pi \mid N \sim G(\tau)$ for some $\tau \in \widehat{N}$, and since $\pi_{\alpha}|N \rightarrow \pi| N$ and $G / N$ is finite, after replacing $\tau$ with some conjugate $\tau^{x}$ if necessary, we can assume that $\tau_{\alpha} \rightarrow \tau$ in $\widehat{N}$. It follows that $S \subseteq G_{\tau}$ and $\pi \in \widehat{G}_{G_{\tau}}$.

Let $|\mathcal{S}|=r$ and write $\mathcal{S}=\left\{S_{1}, \ldots, S_{r}\right\}$ so that $\left[S_{k}: N\right]$ is decreasing with $k$. By the preceding paragraph, $\bigcup_{j=1}^{k} \widehat{G}_{S_{j}}$ is closed in $\widehat{G}$ for every $1 \leq k \leq r$.

Now, let $C$ be a compact subset of $\widehat{G}_{d}$ and set $E_{j}=C \cap \widehat{G}_{S_{j}}, 1 \leq j \leq r$. The sets $E_{j}$ are pairwise either disjoint or equal since for $S, T \in \mathcal{S}$ either $\widehat{G}_{S} \cap \widehat{G}_{T}=\emptyset$ or $\widehat{G}_{S}=\widehat{G}_{T}$ (which happens precisely when $S$ and $T$ are conjugate). Then $C=$ $\bigcup_{j=1}^{r} E_{j}$, and for every $1 \leq k \leq r, \bigcup_{j=1}^{k} E_{j}$ is closed in $C$ and hence $E_{k}$ is open in $\bigcup_{j=1}^{k} E_{j}$.

We prove by induction on $k$ that

$$
\operatorname{dim}\left(\bigcup_{j=1}^{k} E_{j}\right) \leq \operatorname{rank} N .
$$

This is clear for $k=1$ since $E_{1}$ is homeomorphic to a compact subset of $\left(\widehat{S_{1}}\right)_{S_{1}}$. Let $k \geq 2$ and suppose that the statement holds for $k-1$. We now apply [23] Chapter 3, Proposition 5.6], taking $X$ to be the normal space $\bigcup_{j=1}^{k} E_{j}, n=\operatorname{rank} N$ and $A=\bigcup_{j=1}^{k-1} E_{j}$. We have to check that $\operatorname{dim} F \leq \operatorname{rank} N$ for every closed subset $F$ of $\bigcup_{j=1}^{k} E_{j}$ such that $F \cap\left(\bigcup_{j=1}^{k-1} E_{j}\right)=\emptyset$. Such an $F$ is a compact subset of $\widehat{G}_{d}$ and hence is closed in $\widehat{G}$ [9, Corollary 1 of Section 10]. Since $\widehat{G}_{S_{k}}$ is homeomorphic to $\left(\widehat{S_{k}}\right)_{S_{k}}, \operatorname{dim} F \leq \operatorname{rank} N$ by Lemma 3.2 .

Theorem 3.4. If $G$ is Moore group, then

(i) $\left[\frac{1}{\left[G: G_{F}\right.}\left\lfloor\frac{1}{2} \operatorname{rank}\left(G_{F}\right)\right\rfloor\right]+1 \leq \operatorname{sr}\left(C^{*}(G)\right) \leq \operatorname{sr}\left(C^{*}\left(G_{F}\right)\right)=\left\lfloor\frac{1}{2} \operatorname{rank}\left(G_{F}\right)\right\rfloor+1$.

(ii) $\left\lceil\frac{\operatorname{rank}\left(G_{F}\right)}{2\left[G: G_{F}\right]-1}\right\rceil \leq \operatorname{RR}\left(C^{*}(G)\right) \leq \operatorname{RR}\left(C^{*}\left(G_{F}\right)\right)=\operatorname{rank}\left(G_{F}\right)$. 
Proof. Let $K=\left[G_{F}, G_{F}\right]$. The lower estimates are simple. Indeed, if $J$ is as in Lemma 3.1, then by Lemma 1.1(a) and Lemma 3.1

$$
\begin{aligned}
\operatorname{sr}\left(C^{*}(G)\right) & \geq \operatorname{sr}\left(C^{*}(G) / J\right)=\left\lceil\frac{1}{\left[G: G_{F}\right]}\left\lfloor\frac{1}{2} \operatorname{dim}\left(\left(C^{*}(G) / J\right)^{\wedge}\right)\right\rfloor+1\right. \\
& =\left\lceil\frac{1}{\left[G: G_{F}\right]}\left\lfloor\frac{1}{2} \operatorname{rank}\left(G_{F} / K\right)\right\rfloor+1,\right.
\end{aligned}
$$

and similarly for the real rank.

To establish the upper estimates for the ranks of $C^{*}(G)$, it suffices to show that

$$
\operatorname{sr}\left(C^{*}(G)\right) \leq\left\lfloor\frac{1}{2} \operatorname{rank}\left(G_{F}\right)\right\rfloor+1 \text { and } \operatorname{RR}\left(C^{*}(G)\right) \leq \operatorname{rank}\left(G_{F}\right)
$$

since, by Theorem 2.6,

$$
\operatorname{sr}\left(C^{*}\left(G_{F}\right)\right)=\left\lfloor\frac{1}{2} \operatorname{rank}\left(G_{F}\right)\right\rfloor+1 \text { and } \operatorname{RR}\left(C^{*}\left(G_{F}\right)\right)=\operatorname{rank}\left(G_{F}\right) .
$$

For any irreducible representation $\sigma$ of $K$, let

$$
A_{\sigma}=\operatorname{ind}_{K}^{G} \sigma\left(C^{*}(G)\right) .
$$

Then, by Lemma 2.2 , since $G$ is amenable,

$$
\operatorname{sr}\left(C^{*}(G)\right)=\sup _{\sigma \in \widehat{K}} \operatorname{sr}\left(A_{\sigma}\right) \text { and } \operatorname{RR}\left(C^{*}(G)\right)=\sup _{\sigma \in \widehat{K}} \operatorname{RR}\left(A_{\sigma}\right) .
$$

We claim that each $A_{\sigma}$ is subhomogeneous and that

$$
\operatorname{sr}\left(A_{\sigma}\right) \leq\left\lfloor\frac{1}{2} \operatorname{rank}\left(G_{F}\right)\right\rfloor+1 \text { and } \operatorname{RR}\left(A_{\sigma}\right) \leq \operatorname{rank}\left(G_{F}\right) .
$$

To that end, fix $\sigma \in \widehat{K}$, set $A=A_{\sigma}$ and notice first that

$$
\widehat{A}=\operatorname{supp}\left(\operatorname{ind}_{K}^{G} \sigma\right)=\{\pi \in \widehat{G}: \pi \mid K \geq \sigma\} .
$$

Now, for every $\pi \in \widehat{A}$, there exists $\tau \in \widehat{G_{F}}$ such that $\pi \mid G_{F} \geq \tau$ and $\tau \mid K \geq \sigma$. Indeed, this follows from the fact that $\pi \mid G_{F}$ is a multiple of the direct sum of representations in some $G$-orbit in $\widehat{G_{F}}$ and that $\pi \mid K$ is a multiple of the direct sum of all the representations in $G(\sigma)$. Let $\pi, \pi_{0} \in \widehat{A}$ and let $\tau, \tau_{0} \in \widehat{G_{F}}$ be as above. Since $G_{F}$ is a type I group with relatively compact conjugacy classes and $\tau\left|K \sim \tau_{0}\right| K$, it follows that $\tau=\tau_{0} \otimes \chi$ for some character $\chi$ of $G_{F} / K$ (see [13. Theorem 2]). This implies that

$$
\begin{aligned}
\operatorname{dim} \pi & \leq \operatorname{dim}\left(\operatorname{ind}_{K}^{G} \tau\right)=\left[G: G_{F}\right] \operatorname{dim} \tau \\
& =\left[G: G_{F}\right] \operatorname{dim} \tau_{0} \leq\left[G: G_{F}\right] \operatorname{dim} \pi_{0} .
\end{aligned}
$$

This shows that $A$ is subhomogeneous.

Let $m$ be the maximal dimension of irreducible representations of $A$, and let $I_{d}$ $(0 \leq d \leq m)$ be defined as in Lemma 1.4, so that

$$
A=I_{0} \supseteq I_{1} \supseteq \ldots \supseteq I_{m}=\{0\}
$$

and ${\widehat{I_{d-1} / I_{d}}}=\widehat{A}_{d}$. Then, by Lemma 1.3 ,

$$
\operatorname{sr}(A)=\max _{1 \leq d \leq m} \operatorname{sr}\left(I_{d-1} / I_{d}\right) \text { and } \operatorname{RR}(A)=\max _{1 \leq d \leq m} \operatorname{RR}\left(I_{d-1} / I_{d}\right) .
$$


Fix $1 \leq d \leq m$ and let $\mathcal{C}$ denote the set of all compact subsets of $\widehat{I_{d-1} / I_{d}}$. Since ${\widehat{I} d-1 / I_{d}}=\widehat{A}_{d} \subseteq \widehat{G}_{d}$, Proposition 1.2 and Proposition 3.3 yield that

$$
\operatorname{sr}\left(I_{d-1} / I_{d}\right)=\sup _{C \in \mathcal{C}}\left\lceil\frac{1}{d}\left\lfloor\frac{1}{2} \operatorname{dim} C\right\rfloor\right\rceil+1 \leq\left\lfloor\frac{1}{2} \operatorname{dim} G_{F}\right\rfloor+1
$$

and

$$
\operatorname{RR}\left(I_{d-1} / I_{d}\right)=\sup _{C \in \mathcal{C}}\left\lceil\frac{\operatorname{dim} C}{2 d-1}\right\rceil \leq \operatorname{rank}\left(G_{F}\right) .
$$

This finishes the proof of the theorem.

Corollary 3.5. For a Moore group $G$ the following three conditions are equivalent:

(i) $\operatorname{sr}\left(C^{*}(G)\right)<\infty$.

(ii) $\operatorname{RR}\left(C^{*}(G)\right)<\infty$.

(iii) $G_{F}$ has finite rank.

Proof. (i) $\Rightarrow$ (ii) is clear, and (ii) $\Rightarrow$ (iii) as well as (iii) $\Rightarrow$ (i) is an immediate consequence of Theorem 3.4 .

Let $G$ be a locally compact group of bounded representation degree and let $A$ be an abelian closed normal subgroup of finite index in $G$. Since $A \subseteq G_{F}$ and $\operatorname{rank} A$ $=\operatorname{rank}\left(G_{F}\right)$, by Theorem 3.4

$$
\left\lceil\frac{1}{[G: A]}\left\lfloor\frac{1}{2} \operatorname{rank} A\right\rfloor \mid+1 \leq \operatorname{sr}\left(C^{*}(G)\right) \leq \operatorname{sr}\left(C^{*}(A)\right)=\left\lfloor\frac{1}{2} \operatorname{rank} A\right\rfloor+1\right.
$$

and

$$
\left\lceil\frac{\operatorname{rank} A}{2[G: A]-1}\right\rceil \leq \operatorname{RR}\left(C^{*}(G)\right) \leq \operatorname{RR}\left(C^{*}(A)\right)=\operatorname{rank} A .
$$

The first of these inequalities has been obtained earlier for second countable groups by Schulz [25, Corollary 3.3] as an application of a result on the stable rank of certain crossed product $C^{*}$-algebras.

\section{Groups With FInite-Dimensional IRREDUCiBle REPRESENTATIONS: ATtAINMENT OF THE UPPER BOUNDS FOR THE RANKS}

In this section we mainly concentrate on the question of when $\operatorname{sr}\left(C^{*}(G)\right)=$ $\operatorname{sr}\left(C^{*}\left(G_{F}\right)\right)$ and $\operatorname{RR}\left(C^{*}(G)\right)=\operatorname{RR}\left(C^{*}\left(G_{F}\right)\right)$.

We first observe that $\operatorname{RR}\left(C^{*}(G)\right)=\operatorname{RR}\left(C^{*}\left(G_{F}\right)\right)$ implies that $\operatorname{sr}\left(C^{*}(G)\right)=$ $\operatorname{sr}\left(C^{*}\left(G_{F}\right)\right)$. Indeed, by Theorem 3.4

$$
\begin{aligned}
\frac{1}{2}\left\{\operatorname{rank}\left(G_{F}\right)+1\right\} & =\frac{1}{2}\left\{\operatorname{RR}\left(C^{*}\left(G_{F}\right)\right)+1\right\}=\frac{1}{2}\left\{\operatorname{RR}\left(C^{*}(G)\right)+1\right\} \\
& \leq \operatorname{sr}\left(C^{*}(G)\right) \leq \operatorname{sr}\left(C^{*}\left(G_{F}\right)\right)=\left\lfloor\frac{1}{2} \operatorname{rank}\left(G_{F}\right)\right\rfloor+1 .
\end{aligned}
$$

Exploiting this inequality in the two cases that $\operatorname{rank}\left(G_{F}\right)$ is even or odd readily shows that $\operatorname{sr}\left(C^{*}(G)\right)=\operatorname{sr}\left(C^{*}\left(G_{F}\right)\right)$.

Lemma 4.1. Let $G$ be a Moore group and suppose that the quotient group $G / G_{F}^{c}$ has a finite commutator subgroup. Then

$$
\operatorname{sr}\left(C^{*}(G)\right)=\operatorname{sr}\left(C^{*}\left(G_{F}\right)\right) \text { and } \operatorname{RR}\left(C^{*}(G)\right)=\operatorname{RR}\left(C^{*}\left(G_{F}\right)\right) .
$$


Proof. By the preceding remark it suffices to establish the real rank assertion. Recall that, by Theorem 2.6, $\operatorname{RR}\left(C^{*}\left(G_{F}\right)\right)=\operatorname{RR}\left(C^{*}\left(G_{F} / G_{F}^{c}\right)\right)$. Notice next that since $G_{F}$ is of finite index in $G$ and $G / G_{F}^{c}$ has a finite commutator subgroup, $\operatorname{RR}\left(C^{*}\left(G_{F} / G_{F}^{c}\right)\right)=\operatorname{RR}\left(C^{*}\left(G / G_{F}^{c}\right)\right)$ by Corollary 2.9. Thus it remains to show that $\operatorname{RR}\left(C^{*}(G)\right)=\operatorname{RR}\left(C^{*}\left(G / G_{F}^{c}\right)\right)$, and for that it is enough to show that $\operatorname{RR}\left(C^{*}(G)\right) \leq \operatorname{RR}\left(C^{*}\left(G / G_{F}^{c}\right)\right)$.

Assume first that $G$ is compactly generated. Then $G_{F}$ is compactly generated as well, and hence $G_{F}^{c}$ is compact. Define a subgroup $K$ of $G$ by $K \supseteq G_{F}^{c}$ and $K / G_{F}^{c}$ equals the commutator subgroup of $G / G_{F}^{c}$. Then $K$ is a compact normal subgroup of $G$ and $G / K$ is abelian. In particular, $G$ is a group with relatively compact conjugacy classes and hence

$$
\operatorname{RR}\left(C^{*}(G)\right)=\operatorname{rank}\left(G / G^{c}\right)
$$

by Theorems 2.5 and 2.6 .

Now drop the hypothesis that $G$ is compactly generated, and let $\mathcal{H}$ denote the collection of all compactly generated open subgroups of $G$. Then, for every $H \in$ $\mathcal{H}, H / H_{F}^{c}$ is a quotient of $H /\left(H \cap G_{F}^{c}\right)=H G_{F}^{c} / G_{F}^{c}$. Since $G / G_{F}^{c}$ has a finite commutator subgroup, so does $H / H_{F}^{c}$. Thus, by the last paragraph,

$$
\operatorname{RR}\left(C^{*}(H)\right)=\operatorname{rank}\left(H / H^{c}\right)
$$

for each $H \in \mathcal{H}$. This implies

$$
\begin{aligned}
\operatorname{RR}\left(C^{*}(G)\right) & \leq \sup _{H \in \mathcal{H}} \operatorname{RR}\left(C^{*}(H)\right)=\sup _{H \in \mathcal{H}} \operatorname{rank}\left(H / H^{c}\right) \\
& \leq \sup _{H \in \mathcal{H}} \operatorname{rank}\left(H /\left(H \cap G_{F}\right)^{c}\right)=\sup _{H \in \mathcal{H}} \operatorname{rank}\left(H /\left(H \cap G_{F}^{c}\right)\right) .
\end{aligned}
$$

Let $N$ denote the pullback to $G$ of the finite commutator subgroup of $G / G_{F}^{c}$. Then, since $G / N$ is abelian and $N / G_{F}^{c}$ is finite, we obtain that

$$
\begin{aligned}
\operatorname{RR}\left(C^{*}(G)\right) & \leq \sup _{H \in \mathcal{H}} \operatorname{rank}\left(H / H \cap G_{F}^{c}\right)=\sup _{H \in \mathcal{H}} \operatorname{rank}(H / H \cap N) \\
& =\sup _{H \in \mathcal{H}} \operatorname{rank}(H N / N)=\operatorname{rank}(G / N)=\operatorname{RR}\left(C^{*}(G / N)\right) \\
& =\operatorname{RR}\left(C^{*}\left(\left(G / G_{F}^{c}\right) /\left(N / G_{F}^{c}\right)\right)\right) \leq \operatorname{RR}\left(C^{*}\left(G / G_{F}^{c}\right)\right),
\end{aligned}
$$

as required.

The reader might have observed that in the proof of Lemma 4.1 we have only used that $G / G_{F}^{c}$ has a relatively compact commutator subgroup. However, this compactness condition already forces the finiteness of the commutator subgroup of $G / G_{F}^{c}$. In fact, this can easily be seen by using the fact that $G_{F}$ is a direct product $G_{F}=V \times L$, where $V$ is a vector group and $L$ contains a compact open subgroup.

In the next lemma, we shall need the fact that if $N$ is a closed normal subgroup of an $[\mathrm{FC}]^{-}$group $G$ such that $\operatorname{rank}(G / N)=\operatorname{rank} G<\infty$, then $N \subseteq G^{c}$. To see this, let $q: G \rightarrow G / N$ be the quotient homomorphism and let $H=q^{-1}\left((G / N)^{c}\right)$. Then $G^{c} \subseteq H, G / H=(G / N) /(G / N)^{c}$ is compact-free and

$$
\operatorname{rank}\left(G / G^{c}\right)=\operatorname{rank} G=\operatorname{rank}(G / N)=\operatorname{rank}(G / H) .
$$

Since $N \subseteq H$, it suffices to show that $H \subseteq G^{c}$. Thus, after passing to $G / G^{c}$ and $H / G^{c}$, it remains to show that if $B$ is a closed subgroup of a compact-free abelian group $A$ such that $A / B$ is compact-free and $\operatorname{rank}(A / B)=\operatorname{rank} A<\infty$, then $B$ is trivial. 
Let $A=\mathbb{R}^{l} \times D$ and $A / B=\mathbb{R}^{m} \times E$, where $l, m \in \mathbb{N}_{0}$ and $D$ and $E$ are torsion-free discrete abelian groups. The quotient homomorphism $q: A \rightarrow A / B$ maps $\mathbb{R}^{l}$ linearly onto $\mathbb{R}^{m}$ and induces a homomorphism $\bar{q}$ of $A / \mathbb{R}^{l}=D$ onto $(A / B) / \mathbb{R}^{m}=E$. It follows that $m \leq l$ and $\operatorname{rank} E \leq \operatorname{rank} D$. Since, by assumption, $m+\operatorname{rank} E=l+\operatorname{rank} D$, we conclude that $m=l$ and $\operatorname{rank} E=\operatorname{rank} D$. The last equality, together with an elementary argument involving independence, leads to the injectivity of $\bar{q}$. Since $m=l$, the restriction of $q$ to $\mathbb{R}^{l}$ is also injective. Hence $q$ is injective, as required.

Lemma 4.2. Let $G$ be a Moore group.

(i) If $\operatorname{RR}\left(C^{*}\left(G_{F}\right)\right)=\operatorname{RR}\left(C^{*}(G /[G, G])\right)<\infty$, then the quotient group $G / G_{F}^{c}$ has a finite commutator subgroup.

(ii) If $\operatorname{sr}\left(C^{*}\left(G_{F}\right)\right)=\operatorname{sr}\left(C^{*}(G /[G, G])\right)<\infty$, then either $G / G_{F}^{c}$ has a finite commutator subgroup or $\operatorname{rank}\left(G_{F}\right)$ is odd and $\operatorname{rank}\left(G_{F} \cap[G, G]\right)=1$.

Proof. (i) Since $G_{F} / G_{F} \cap[G, G]=G_{F}[G, G] /[G, G]$ is of finite index in $G /[G, G]$, the hypothesis yields that

$$
\begin{aligned}
\operatorname{rank}\left(G_{F}\right) & =\operatorname{RR}\left(C^{*}\left(G_{F}\right)\right)=\operatorname{RR}\left(C^{*}(G /[G, G])\right) \\
& =\operatorname{rank}(G /[G, G])=\operatorname{rank}\left(G_{F} / G_{F} \cap[G, G]\right) .
\end{aligned}
$$

By the remark preceding this lemma, $G_{F} \cap[G, G] \subseteq G_{F}^{c}$. Thus, algebraically,

$$
G_{F}^{c}[G, G] / G_{F}^{c}=[G, G] /\left(G_{F}^{c} \cap[G, G]\right)=[G, G] /\left(G_{F} \cap[G, G]\right) .
$$

Since $G_{F}$ has finite index in $G$, it follows that $G_{F}^{c}$ has finite index in $G_{F}^{c}[G, G]$. Thus, since $G / G_{F}^{c}[G, G]$ is abelian, $G / G_{F}^{c}$ has a finite commutator subgroup.

(ii) The hypothesis implies that

$$
\begin{aligned}
\left\lfloor\frac{1}{2} \operatorname{rank}\left(G_{F}\right)\right\rfloor+1 & =\operatorname{sr}\left(C^{*}\left(G_{F}\right)\right)=\operatorname{sr}\left(C^{*}(G /[G, G])\right) \\
& =\left\lfloor\frac{1}{2} \operatorname{rank}(G /[G, G])\right\rfloor+1 \\
& =\left\lfloor\frac{1}{2} \operatorname{rank}\left(G_{F} / G_{F} \cap[G, G]\right)\right\rfloor+1 .
\end{aligned}
$$

Distinguishing the two cases that $\operatorname{rank}\left(G_{F}\right)$ is even or odd, the statement now follows as in (i).

Theorem 4.3. Let $G$ be a Moore group. Then the following two conditions are equivalent:

(i) $\operatorname{RR}\left(C^{*}(G)\right)=\operatorname{RR}\left(C^{*}\left(G_{F}\right)\right)$.

(ii) Either $\operatorname{rank}\left(G_{F}\right)=1$, or $G / G_{F}^{c}$ has a finite commutator subgroup, or $\operatorname{rank}\left(G_{F}\right)$ $=\infty$.

Proof. (i) $\Rightarrow$ (ii) Suppose that $\operatorname{rank}\left(G_{F}\right)<\infty$. It follows from the proofs of Lemma 2.2 and Theorem 3.4 that $C^{*}(G)$ is a $c_{0}$-direct sum of subhomogeneous $C^{*}$-algebras. By Corollary 3.5 and Lemma 1.4(i) there is a $d$-homogeneous subquotient $I_{d-1} / I_{d}$ of $C^{*}(G)$ such that $\operatorname{RR}\left(C^{*}(G)\right)=\operatorname{RR}\left(I_{d-1} / I_{d}\right)$.

Assume first that $d=1$. Then $I_{0} / I_{1}=C^{*}(G /[G, G])$ and hence $\operatorname{RR}\left(C^{*}\left(G_{F}\right)\right)=$ $\operatorname{RR}\left(C^{*}(G /[G, G])\right)$. Then Lemma $4.2(\mathrm{i})$ shows that $G / G_{F}^{c}$ has a finite commutator subgroup. Otherwise, for some $d>1$,

$$
\operatorname{RR}\left(C^{*}(G)\right)=\sup _{C \in \mathcal{C}}\left\lceil\frac{\operatorname{dim} C}{2 d-1}\right\rceil
$$


(Proposition 1.2), where $\mathcal{C}$ denotes the set of all compact subsets of $\widehat{G}_{d}$. Since $\operatorname{dim} C \leq \operatorname{rank}\left(G_{F}\right)$ by Proposition 3.3, it follows from (i) that

$$
\operatorname{rank}\left(G_{F}\right)=\operatorname{RR}\left(C^{*}\left(G_{F}\right)\right)=\operatorname{RR}\left(C^{*}(G)\right) \leq\left\lceil\frac{\operatorname{rank}\left(G_{F}\right)}{2 d-1}\right\rceil .
$$

This of course implies that $\operatorname{rank}\left(G_{F}\right) \leq 1$, and if $\operatorname{rank}\left(G_{F}\right)=0$, then $G / G_{F}^{c}=$ $G / G_{F}$ is finite.

(ii) $\Rightarrow$ (i) If $G / G_{F}^{c}$ has a finite commutator group, then $\operatorname{RR}\left(C^{*}(G)\right)=$ $\operatorname{RR}\left(C^{*}\left(G_{F}\right)\right)$ by Lemma 4.1. If $\operatorname{rank}\left(G_{F}\right)=1$, then $\operatorname{RR}\left(C^{*}(G)\right) \leq \operatorname{RR}\left(C^{*}\left(G_{F}\right)\right)=$ 1. On the other hand, since $\left(G_{F} / G_{F}^{c}\right)^{c}$ is trivial and $G_{F} / G_{F}^{c}$ is non-trivial, $\operatorname{RR}\left(C^{*}\left(G / G_{F}^{c}\right)\right) \geq 1$ [14, Lemma 2].

Theorem 4.4. Let $G$ be a Moore group. Then the following two conditions are equivalent:

(i) $\operatorname{sr}\left(C^{*}(G)\right)=\operatorname{sr}\left(C^{*}\left(G_{F}\right)\right)$.

(ii) Either $\operatorname{rank}\left(G_{F}\right) \leq 3$, or $G / G_{F}^{c}$ has a finite commutator subgroup, or $\operatorname{rank}\left(G_{F}\right)$ is odd and $\operatorname{rank}\left(G_{F} \cap[G, G]\right)=1$, or $\operatorname{rank}\left(G_{F}\right)=\infty$.

Proof. (i) $\Rightarrow$ (ii) Suppose that $\operatorname{rank}\left(G_{F}\right)<\infty$. By Corollary 3.5 and Lemma 1.4(ii) (cf. the proof of Theorem 4.3), there exists a $d$-homogeneous subquotient $I_{d-1} / I_{d}$ of $C^{*}(G)$ such that $\operatorname{sr}\left(C^{*}(G)\right)=\operatorname{sr}\left(I_{d-1} / I_{d}\right)$. By Propositions 1.2 and 3.3 we have

$$
\left\lfloor\frac{1}{2} \operatorname{rank}\left(G_{F}\right)\right\rfloor+1=\operatorname{sr}\left(C^{*}\left(G_{F}\right)\right)=\operatorname{sr}\left(C^{*}(G)\right) \leq\left\lceil\frac{1}{d}\left\lfloor\frac{1}{2} \operatorname{rank}\left(G_{F}\right)\right\rfloor\right\rceil+1 .
$$

If $d>1$, this inequality implies that $\operatorname{rank}\left(G_{F}\right) \leq 3$.

(ii) $\Rightarrow$ (i) If $G / G_{F}^{c}$ has a finite commutator subgroup, then (i) holds by Lemma 4.1. Next suppose that $\operatorname{rank}\left(G_{F}\right)=2 k+1$ and $\operatorname{rank}\left(G_{F} \cap[G, G]\right)=1$. Then

$$
\begin{aligned}
\operatorname{sr}\left(C^{*}(G)\right) & \geq \operatorname{sr}\left(C^{*}(G /[G, G])\right)=\left\lfloor\frac{1}{2} \operatorname{rank}(G /[G, G])\right\rfloor+1 \\
& =\left\lfloor\frac{1}{2} \operatorname{rank}\left(G_{F} / G_{F} \cap[G, G]\right)\right\rfloor+1=k+1 \\
& =\left\lfloor\frac{1}{2} \operatorname{rank}\left(G_{F}\right)\right\rfloor+1=\operatorname{sr}\left(C^{*}\left(G_{F}\right)\right) .
\end{aligned}
$$

Since $\operatorname{sr}\left(C^{*}(G)\right) \leq \operatorname{sr}\left(C^{*}\left(G_{F}\right)\right)$, (i) follows.

If $\operatorname{rank}\left(G_{F}\right)=1$, then $\operatorname{sr}\left(C^{*}\left(G_{F}\right)\right)=1$ and hence $\operatorname{sr}\left(C^{*}(G)\right)=1$.

Finally, if $2 \leq \operatorname{rank}\left(G_{F}\right) \leq 3$, then $\operatorname{sr}\left(C^{*}\left(G_{F}\right)\right)=\left\lfloor\frac{1}{2} \operatorname{rank}\left(G_{F}\right)\right\rfloor+1=2$ and, with the notation of Lemma 3.1,

$$
\begin{aligned}
\operatorname{sr}\left(C^{*}(G)\right) & \geq \operatorname{sr}\left(C^{*}(G) / J\right)=\left\lceil\frac{1}{\left[G: G_{F}\right]}\left\lfloor\frac{1}{2} \operatorname{rank}\left(G_{F} / K\right)\right\rfloor+1\right. \\
& =\left\lceil\frac{1}{\left[G: G_{F}\right]}\left\lfloor\frac{1}{2} \operatorname{rank}\left(G_{F}\right)\right\rfloor \mid+1=2,\right.
\end{aligned}
$$

because $K$ is compact. Thus $\operatorname{sr}\left(C^{*}(G)\right)=2$.

When $G$ is of bounded representation dimension and $A$ is an abelian closed normal subgroup of finite index in $G$, then in all the preceding results $G_{F}$ can be replaced with $A$. Moreover, the condition that $G / A^{c}$ has a finite commutator subgroup is equivalent to $G / A^{c}$ having a centre of finite index. This can be seen as follows. 
Let $H$ be a locally compact group with finite conjugacy classes, and suppose that $H$ has an abelian normal subgroup $C$ of finite index. Then the centre of $H$ has finite index in $H$. Indeed, if $F$ is a finite subset of $H$ such that $H=F C$, then $C_{H}(F)$, the centralizer of $F$ in $H$, is of finite index in $H$ and hence $C \cap C_{H}(F)$ has finite index in $H$ and is contained in the centre of $H$ because $C$ is abelian.

A particularly interesting special class, including the so-called crystal groups, is provided by those discrete groups which contain a normal subgroup of finite index that is isomorphic to $\mathbb{Z}^{m}$ for some $m \in \mathbb{N}$. For such groups Theorems 4.3 and 4.4 can be reformulated as follows.

Corollary 4.5. Let $G$ contain a normal subgroup $A$ of finite index such that $A$ is isomorphic to $\mathbb{Z}^{m}$ for some $m \in \mathbb{N}$. Then

(i) $\operatorname{RR}\left(C^{*}(G)\right)=m$ if and only if either $m=1$ or $A$ is contained in the centre of $G$,

(ii) $\operatorname{sr}\left(C^{*}(G)\right)=\left\lfloor\frac{m}{2}\right\rfloor+1$ if and only if either $m \leq 3$ or $A$ is contained in the centre of $G$ or $m$ is odd and $A \cap[G, G]$ is infinite cyclic.

Proof. In view of Theorems 4.3 and 4.4 it only remains to show that if the centre $Z(G)$ has finite index in $G$ and $A=\mathbb{Z}^{m}$, then $A$ is contained in the centre of $G$. Since $A \cap Z(G)$ has finite index in $G$ and $A=\mathbb{Z}^{m}, A \cap Z(G)$ contains $q \mathbb{Z}^{m}$ for some $q \in \mathbb{N}$. Now, every automorphism of $\mathbb{Z}^{m}$ is given by the action of a matrix in $G L(m, \mathbb{Z})$ and if such a matrix acts trivially on $q \mathbb{Z}^{m}$, then it does so on $\mathbb{Z}^{m}$. Thus $A$ is contained in the centre of $G$.

We continue with a few illustrative examples.

Example 4.6. (1) Let $n \in \mathbb{N}, n \geq 2$, and let $\gamma$ denote the automorphism of $\mathbb{Z}^{n}$ defined by

$$
\gamma(x)=\left(x_{n}, x_{1}, \ldots, x_{n-1}\right)\left(x=\left(x_{1}, \ldots, x_{n}\right) \in \mathbb{Z}^{n}\right) .
$$

Let $\Gamma$ be the cyclic group of order $n$ generated by $\gamma$ and form the associated semidirect product $G=\Gamma \ltimes \mathbb{Z}^{n}$. We claim that

$$
\operatorname{RR}\left(C^{*}(G)\right)=1, \operatorname{sr}\left(C^{*}(G)\right)=2 \text { and } \operatorname{dim} \widehat{G} \geq n-1 .
$$

Thus $C^{*}(G)$ serves as an example, in the context of group $C^{*}$-algebras, of the kind mentioned at the end of Section 1.

The dual action of $\Gamma$ on $\widehat{\mathbb{Z}^{n}}=\mathbb{T}^{n}$ is given by $\gamma(z)=\left(z_{n}, z_{1}, \ldots, z_{n-1}\right)$ for $z=\left(z_{1}, \ldots, z_{n}\right) \in \mathbb{T}^{n}$. The subgroups of $\Gamma$ are precisely the groups $\Gamma_{d}$, generated by $\gamma^{d}$, where $d \mid n$. Let

$$
X_{d}=\left\{z \in \mathbb{T}^{n}: \gamma^{d}(z)=z \text { and } \gamma^{k}(z) \neq z \text { for } k<d\right\} .
$$

If $n=d m$, then straightforward calculations show that $\gamma^{d}(z)=z$ if and only if, for any $1 \leq l \leq d, z_{l}=z_{d+l}=\ldots=z_{(m-1) d+l}$. Thus $X_{d}$ is homeomorphic to the set

$$
\left\{\left(w_{1}, \ldots, w_{d}\right) \in \mathbb{T}^{d}: w_{i} \neq w_{j} \text { for } i \neq j\right\} .
$$

Now, by Mackey's theory,

$$
\widehat{G}_{d}=\left\{\operatorname{ind}_{\Gamma_{d} \ltimes \mathbb{Z}^{n}}^{G}(\beta \alpha): \alpha \in \widehat{\mathbb{Z}^{n}}, G_{\alpha}=\Gamma_{d} \ltimes \mathbb{Z}^{n}, \beta \in \widehat{\Gamma_{d}}\right\} .
$$

So $\widehat{G}_{d}$ is a disjoint union of open sets each of which is homeomorphic to $X_{d}$. It follows that $d$ is the maximal dimension of compact subsets of $\widehat{G}_{d}$. Hence

$$
\operatorname{RR}\left(C^{*}(G)\right)=\max _{d \mid n}\left\lceil\frac{d}{2 d-1}\right\rceil=1
$$


and

$$
\operatorname{sr}\left(C^{*}(G)\right)=1+\max _{d \mid n}\left\lceil\frac{1}{d}\left\lfloor\frac{1}{2} d\right\rfloor\right\rceil=2 .
$$

Finally, that $\operatorname{dim} \widehat{G} \geq n-1$ is shown in exactly the same way as in the example at the end of Section 1 by considering a sequence in $X_{n}$ converging to the trivial character $(1, \ldots, 1)$ of $\mathbb{Z}^{n}$ and the limit set $\widehat{\Gamma}=\widehat{G / \mathbb{Z}^{n}}$ of the induced sequence in $\widehat{G}$.

(2) It is not difficult to construct examples of discrete groups $G$ such that $G$ fails to be a group with finite conjugacy classes but has an abelian normal subgroup $A$ of finite index such that $G / A^{c}$ is abelian, so that $\operatorname{RR}\left(C^{*}(G)\right)=\operatorname{RR}\left(C^{*}(A)\right)$.

Let $R$ be a commutative ring such that $(R,+)$ is torsion-free, and let $I$ be an ideal of $R$ such that $(R / I,+)$ is a torsion group. Let $S$ be any subgroup of $R$ and let $\phi$ be a finite group of automorphisms of $R$ such that $\varphi(I) \subseteq I$ for all $\varphi \in \phi$. Form the semidirect product $G=\phi \ltimes(S \times R / I)$, where $\varphi \in \phi$ acts on $S \times(R / I)$ by $\varphi \cdot(x, y+I)=(x, y-\varphi(x)+I), x \in S, y \in R$. Of course, $A=S \times(R / I)$ is an abelian normal subgroup of finite index in $G$. Moreover, $A^{c}=R / I$ by hypothesis, and $G /(R / I)$ is abelian.

(3) In Theorems 4.3 and 4.4 we have characterized those Moore groups $G$ for which either of the ranks of $C^{*}(G)$ attains its upper bound. In contrast, there seems to be no reasonable way of characterizing those $G$ for which the lower bounds,

$$
\left\lceil\frac{\operatorname{rank}\left(G_{F}\right)}{2\left[G: G_{F}\right]-1}\right\rceil \text { and }\left\lceil\frac{1}{\left[G: G_{F}\right]}\left\lfloor\frac{1}{2} \operatorname{rank}\left(G_{F}\right)\right\rfloor+1\right. \text {, }
$$

are attained. However, this happens quite often. For simplicity, we present just one class of examples.

Let $G$ be a locally compact group containing an abelian closed normal subgroup $A$ of finite rank and of prime index $p$, and suppose that $G \neq G_{F}$. Then, since $p$ is a prime, $G_{F}=A$ and for any $\chi \in \widehat{A}$, either $G_{\chi}=A$ or $G_{\chi}=G$. Since $G /[A, G]$ has a centre of finite index and $G_{\chi}=G$ precisely when $\chi([A, G])=\{1\}$, the analysis of Sections 2 and 3 shows that

$$
\operatorname{RR}\left(C^{*}(G)\right)=\max \left\{\left\lceil\frac{\operatorname{rank} A}{2 p-1}\right\rceil, \operatorname{rank}(A /[A, G])\right\} .
$$

Now, write $\operatorname{rank} A=n(2 p-1)+r$, where $0 \leq r<2 p-1$. Then $\left\lceil\frac{\operatorname{rank} A}{2 p-1}\right\rceil=n$ if $r=0$ and $=n+1$ if $r>0$. It follows that

$$
\operatorname{RR}\left(C^{*}(G)\right)=\left\lceil\frac{\operatorname{rank} A}{2[G: A]-1}\right\rceil
$$

if and only if $\operatorname{rank}([A, G]) \geq 2 n(p-1)$ if $r=0$ and $\geq 2 n(p-1)+r-1$ if $r>0$. Similarly, it can be shown that

$$
\operatorname{sr}\left(C^{*}(G)\right)=1+\max \left\{\left\lceil\frac{1}{p}\left\lfloor\frac{1}{2} \operatorname{rank} A\right\rfloor\right],\lfloor\operatorname{rank}(A /[A, G])\rfloor\right\} .
$$

Then the condition that

$$
\operatorname{sr}\left(C^{*}(G)\right)=1+\left\lceil\frac{1}{[G: A]}\left\lfloor\frac{1}{2} \operatorname{rank} A\right\rfloor\right\rceil
$$

can also easily be expressed in terms of the rank of $[A, G]$. 


\section{NOTE ADDED IN PROOF}

The authors have recently obtained comparable results for nilpotent groups in On the stable rank and real rank of group $C^{*}$-algebras of nilpotent locally compact groups (Math. Scand., to appear).

\section{REFERENCES}

[1] E.J. Beggs and D.E. Evans, The real rank of algebras of matrix valued functions, Intern. J. Math. 2 (1991), 131-137. MR1094700|(92e:46114)

[2] L.G. Brown and G.K. Pedersen, $C^{*}$-algebras of real rank zero, J. Funct. Anal. 99 (1991), 131-149. MF 1120918 (92m:46086)

[3] K.R. Davidson, $C^{*}$-algebras by example, Fields Institute Monographs, American Mathematical Society, 1996. MR1402012 (97i:46095)

[4] J. Dixmier, $C^{*}$-algebras, North-Holland, 1977. MR0458185|(56:16388)

[5] K.J. Dykema, U. Haagerup and M. Rørdam, The stable rank of some free product $C^{*}$-algebras, Duke Math. J. 90 (1997), 95-121. MR1478545 (99g:46077a)

[6] K.J. Dykema and P. de la Harpe, Some groups whose reduced $C^{*}$-algebras have stable rank one, J. Math. Pures Appl. 78 (1999), 591-608. MR1708667 (2000f:46070)

[7] S. Echterhoff and E. Kaniuth, Certain group extensions and twisted covariance algebras with generalized continuous trace, In: Harmonic Analysis (Eds. P. Eymard and J.P. Pier), LNM 1359, 159-169, Springer-Verlag, 1987. MR0974312 (90c:46083)

[8] N. Elhage Hassan, Rang reel de certaines extensions, Proc. Amer. Math. Soc. 123 (1995), 3067-3073. MR1264814 (95m:46117)

[9] J.M.G. Fell, The dual spaces of $C^{*}$-algebras, Trans. Amer. Math. Soc. 94 (1960), 365-403. MR 0146681 (26:4201)

[10] J.M.G. Fell and R.S. Doran, Representations of *-algebras, locally compact groups, and Banach *-algebraic bundles, Vol. 2, Academic Press, 1988. MR0936629 (90c:46002)

[11] S. Grosser and M. Moskowitz, Compactness conditions in topological groups, J. Reine Angew. Math. 246 (1971), 1-40. MR0284541 (44:1766)

[12] E. Hewitt and K.A. Ross, Abstract harmonic analysis I, Springer-Verlag, 1963. MR0156915 $(28: 158)$

[13] E. Kaniuth, Primitive ideal spaces of groups with relatively compact conjugacy classes, Arch. Math. 32 (1979), 16-24. MR0532844 (81f:22009)

[14] E. Kaniuth, Group $C^{*}$-algebras of real rank zero or one, Proc. Amer. Math. Soc. 119 (1993), 1347-1354. MR.1164146 (94a:46074)

[15] E. Kaniuth and K.F. Taylor, Kazhdan constants and the dual space topology, Math. Ann. 293 (1992), 495-508. MR1170523 (93i:22003)

[16] C.C. Moore, Groups with finite dimensional irreducible representations, Trans. Amer. Math. Soc. 166 (1972), 401-410. MR0302817 (46:1960)

[17] K. Nagami, Dimension theory, Academic Press, New York/London, 1970. MR0271918 $(42: 6799)$

[18] M. Nagisa, Stable rank of some full group $C^{*}$-algebras of groups obtained by the free product, Intern. J. Math. 8 (1997), 375-382. MF.1454479 (98i:46057)

[19] M. Nagisa, H. Osaka and N.C. Phillips, Ranks of algebras of continuous $C^{*}$-algebra valued functions, Canad. J. Math. 53 (2001), 979-1030. MR.1859764 (2002h:46094)

[20] V. Nistor, Stable range for tensor products of extensions of $K$ by $C(X)$, J. Oper. Theory 16 (1986), 387-396. MR0860355 (88b:46085)

[21] V. Nistor, Stable rank for a certain class of type I $C^{*}$-algebras, J. Oper. Theory 17 (1987), 365-373. MF 0887231 (88h:46110)

[22] H. Osaka, Real rank of crossed products by connected compact groups, Bull. London Math. Soc. 27 (1995), 257-264. MR1328702 (96e:46076)

[23] A.R. Pears, Dimension theory of general spaces, Cambridge University Press, Cambridge, 1975. MR0394604 (52:15405)

[24] M.A. Rieffel, Dimension and stable rank in the $K$-theory of $C^{*}$-algebras, Proc. London Math. Soc.(3) 6 (1983), 301-333. MR0693043 (84g:46085)

[25] E. Schulz, The stable rank of crossed products of sectional $C^{*}$-algebras by compact Lie groups, Proc. Amer. Math. Soc. 112 (1991), 733-744. MR1042272 (91j:46082) 
[26] A.J.-L. Sheu, A cancellation theorem for projective modules over the group $C^{*}$-algebras of certain nilpotent Lie groups, Canad. J. Math. 39 (1987), 365-427. MR0899843 (88i:46093)

[27] T. Sudo, Stable rank of the reduced $C^{*}$-algebras of non-amenable Lie groups of type I, Proc. Amer. Math. Soc. 125 (1997), 3647-3654. MR1415371|(98b:46093)

[28] T. Sudo, Stable rank of the $C^{*}$-algebras of amenable Lie groups of type I, Math. Scand. 84 (1999), 231-242. MR 1700530 (2000g:46078)

[29] T. Sudo, Dimension theory of group $C^{*}$-algebras of connected Lie groups of type I, J. Math. Soc. Japan 42 (2000), 583-590. MR1760606 (2001h:22007)

[30] T. Sudo and H. Takai, Stable rank of the $C^{*}$-algebras of nilpotent Lie groups, Intern. J. Math. 6 (1995), 439-446. MR1327158 (96b:46083)

[31] T. Sudo and H. Takai, Stable rank of the $C^{*}$-algebras of solvable Lie groups of type I, J. Oper. Theory 38 (1997), 67-86. MF 1462016 (99a:46125)

[32] J. Tomiyama and M. Takesaki, Applications of fibre bundles to the certain class of $C^{*}$ algebras, Tohoku Math. J. 13 (1963), 498-523. MR0139025 (25:2465)

Department of Mathematical Sciences, University of Aberdeen, Aberdeen AB24 3UE, Scotland, United Kingdom

E-mail address: r.archbold@maths.abdn.ac.uk

Institut für Mathematik, Universität Paderborn, D-33095 Paderborn, Germany

E-mail address: kaniuth@math.uni-paderborn.de 